\title{
A MINIMAL PARTITION PROBLEM WITH TRACE CONSTRAINT IN THE GRUSHIN PLANE.
}

\author{
VALENTINA FRANCESCHI
}

\begin{abstract}
We study a variational problem for the perimeter associated with the Grushin plane, called minimal partition problem with trace constraint. This consists in studying how to enclose three prescribed areas in the Grushin plane, using the least amount of perimeter, under an additional "one-dimensional" constraint on the intersections of their boundaries. We prove existence of regular solutions for this problem, and we characterize them in terms of isoperimetric sets, showing differences with the Euclidean case. The problem arises from the study of quantitative isoperimetric inequalities and has connections with the theory of minimal clusters.
\end{abstract}

\section{INTRODUCTION}

Minimal partition problems are variational problems in which the aim is to find the best configuration of a given number of regions in the space in order to minimize their total perimeter. For a volume measure $V$ and a perimeter measure $\mathcal{P}$ on $\mathbb{R}^{n}, n \in \mathbb{N}$, a cluster is a finite disjoint family of sets $\left\{E_{i}\right\}_{i=1}^{m}, E_{i} \subset \mathbb{R}^{n}$ having finite perimeter $\mathcal{P}$ and finite, positive volume $V$. Given $v_{i}>0$ for $i=1, \ldots, m$, by a minimal partition problem we mean any problem of the form

$$
\inf \left\{\mathscr{P}_{\mathcal{P}}(E): E=E_{1} \cup \cdots \cup E_{m} \subset \mathbb{R}^{2}, V\left(E_{i}\right)=v_{i}\right\}
$$

where $\left\{E_{i}\right\}_{i=1}^{m}$ is a cluster and $\mathscr{P}_{\mathcal{P}}$ is defined by

$$
\mathscr{P}_{\mathcal{P}}(E)=\frac{1}{2}\left\{\mathcal{P}\left(\mathbb{R}^{n} \backslash E\right)+\sum_{i=1}^{m} \mathcal{P}\left(E_{i}\right)\right\}
$$

(cf. [20, 24]). When $\mathcal{P}=P$ is the De Giorgi perimeter and $V=\mathcal{L}^{n}$ is the $n$-dimensional Lebesgue measure, existence of minimizers is proved in [1]. In the 1993 paper [7], the authors provide the first complete solution to a minimal partition problem, in the case when $n=2$, $\mathcal{P}=P, V=\mathcal{L}^{n}$ and $m=2$ : the unique minimizers are the so called double bubbles. This result has been extended to $n \geq 3$ in [19, 27], while for general dimensions and $m \geq 3$, several open questions about minimal clusters in the Euclidean setting are still open (see [24]).

In the present paper, we study a planar minimal partition problem with trace constraint that consists in studying how to enclose three prescribed volumes in $\mathbb{R}^{2}$, using the least amount of perimeter, under an additional 1-dimensional constraint on the intersection of their boundaries, see $(1.2 \mathrm{~b})$. We will consider $V=\mathcal{L}^{2}$ and $\mathcal{P}$ to be the anisotropic perimeter associated with the so called Grushin plane, defined at the end of the introduction, see (1.6).

We set up the minimal partition problem with trace constraint for a perimeter measure $\mathcal{P}$ on $\mathbb{R}^{2}$ and the Lebesgue measure $\mathcal{L}^{2}$, in a class of symmetric sets in $\mathbb{R}^{2}$. We say that a set $E \subset \mathbb{R}^{2}$ is $x$-symmetric (resp. $y$-symmetric) if $(x, y) \in E$ implies $(-x, y) \in E$ (resp. if $(x, y) \in E$ implies $(x,-y) \in E)$. We say that $E$ is $y$-convex if the section $E_{x}=\{y \in \mathbb{R}:(x, y) \in E\}$ is an interval

2010 Mathematics Subject Classification. 49Q20, 53C17.

Key words and phrases. Minimal clusters, Grushin plane, Quantitative Isoperimetric Inequality. 
for every $x \in \mathbb{R}$; finally we say that $E$ is $y$-Schwarz symmetric if it is $y$-symmetric and $y$-convex. We denote by $\mathscr{S}_{x}$ the class of $\mathcal{L}^{2}$-measurable, $x$-symmetric sets in $\mathbb{R}^{2}$ and by $\mathscr{S}_{y}^{*}$ the class of $\mathcal{L}^{2}$-measurable and $y$-Schwarz symmetric sets in $\mathbb{R}^{2}$.

Given $v_{1}, v_{2}, h_{1}, h_{2} \geq 0$, we define the class $\mathcal{A}=\mathcal{A}\left(v_{1}, v_{2}, h_{1}, h_{2}\right)$ of all sets $E \in \mathscr{S}_{x} \cap \mathscr{S}_{y}^{*}$ such that for some $x_{0}>0$, called partitioning point of $E$, the sets

$$
E^{l}=\left\{(x, y) \in E: x<-x_{0}\right\}, \quad E^{c}=\left\{(x, y) \in E:|x|<x_{0}\right\}, \quad E^{r}=\left\{(x, y) \in E: x>x_{0}\right\}
$$

satisfy

$$
\begin{gathered}
\mathcal{L}^{2}\left(E^{c}\right)=v_{1}, \quad \mathcal{L}^{2}\left(E^{l}\right)=\mathcal{L}^{2}\left(E^{r}\right)=v_{2} / 2, \\
{\left[-h_{1}, h_{1}\right] \subset \operatorname{tr}_{x_{0}-}^{x} E,\left[-h_{2}, h_{2}\right] \subset \operatorname{tr}_{x_{0}+}^{x} E,}
\end{gathered}
$$

where $\operatorname{tr}_{x_{0} \pm}^{x} E$ denote the left and right traces of the set $E$ at the point $x_{0}$, introduced in Definition A.2. Choosing $h_{1}=h_{2}=h>0$, the trace constraint $(1.2 \mathrm{~b})$ is a relaxed version of the equality

$$
E_{x_{0}}=E_{-x_{0}}=[-h, h] \text {. }
$$

In other words, a set $E \in \mathcal{A}$ is such that $E^{l}, E^{c}, E^{r}$ have prescribed volumes and $E^{c}$ touches $E^{r}$ and $E^{l}$ in segments of a prescribed length, see Figure 1 .

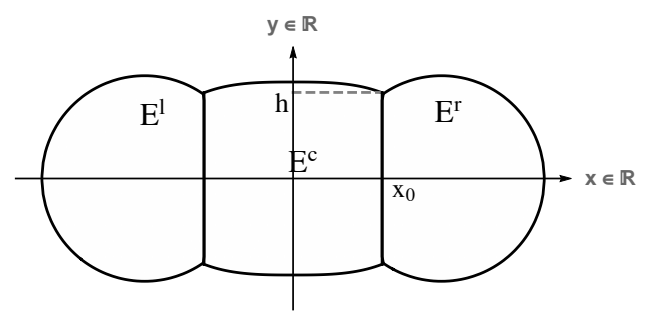

Figure 1. A set in the class $\mathcal{A}$, for $h_{1}=h_{2}=h$.

We study existence of regular solutions to

$$
\inf \left\{\mathscr{P}_{\mathcal{P}}(E): E \in \mathcal{A}\left(v_{1}, v_{2}, h_{1}, h_{2}\right)\right\}, \quad v_{1}, v_{2}, h_{1}, h_{2} \geq 0 .
$$

Our interest in Problem (1.4) comes from the study of the stability of isoperimetric inequalities. In the seminal paper [16], the authors present a symmetrization technique in the Euclidean space $\mathbb{R}^{n}$, to prove existence of a dimensional constant $C(n)>0$ such that any $\mathcal{L}^{n}$-measurable set $E \subset \mathbb{R}^{n}$ satisfies

$$
P(E)-P\left(B\left(0, r_{E}\right)\right) \geq C(n)\left(\min _{x \in \mathbb{R}^{n}} \mathcal{L}^{n}\left(E \triangle B\left(x, r_{E}\right)\right)\right)^{2} .
$$

Here, $B(0, r)=\left\{p=\left(p_{1}, \ldots, p_{n}\right) \in \mathbb{R}^{n}: p_{1}^{2}+\cdots+p_{n}^{2}<r^{2}\right\}$, and the quantity $r_{E} \geq 0$ is chosen to have $\mathcal{L}^{n}(E)=\mathcal{L}^{n}\left(B\left(0, r_{E}\right)\right)$. Such inequality is known as the sharp quantitative isoperimetric inequality in $\mathbb{R}^{n}$, see also [6, 8]. A minimal partition problem for the Euclidean perimeter $\mathcal{P}=P$ under additional constraints is used in [16] to prove (1.5) in a class of symmetric sets.

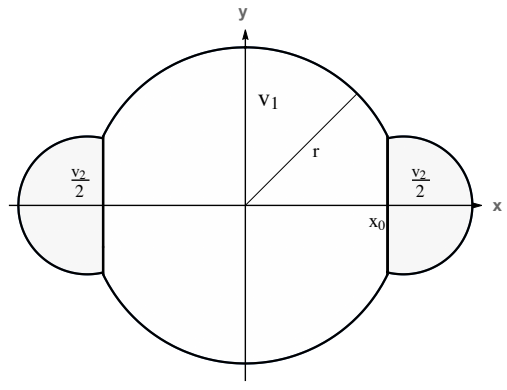

Figure 2. The solution of Problem (1.4) in the Euclidean setting. 
In [16, Lemma 4.3] the authors implicitly use the solution $E$ to problem (1.4) for $\mathcal{P}=P$, with trace constraint given by $(1.3)$. The solution is, for some $x_{0}, r_{0}>0$

$$
E=E^{l} \cup E^{c} \cup E^{r}, \quad E^{c}=B\left(0, r_{0}\right) \cap\left\{(x, y) \in \mathbb{R}^{2}:|x|<x_{0}\right\} .
$$

Notice that the central part $E^{c}$ is the portion of an isoperimetric set lying in a vertical stripe, see Figure 2. This is due to the invariance under translations of the standard perimeter $P$.

In this paper we study the minimal partition problem with trace constraint in the Grushin plane, that is an example of Carnot-Carathéodory space, introduced in the context of hypoelliptic operators by Franchi and Lanconelli in [11. Carnot-Carathéodory spaces are metric spaces in which the distance is defined in association with a family of vector fields $\mathcal{X}=\left\{X_{1}, \ldots, X_{r}\right\}$ on a $n$-dimensional manifold, $n \geq r$. The theory of perimeters in such spaces has been developed starting from the 1990s in [4, 12, 18, and isoperimetric inequalities in Carnot-Carathéodory spaces are a current object of investigation, see [26, 25, 18, 9, 10]. Given $\alpha \geq 0$, the Grushin plane is defined endowing $\mathbb{R}^{2}$ with the family of vector fields $\mathcal{X}_{\alpha}=\left\{\partial_{x},|x|^{\alpha} \partial_{y}\right\}$, where $(x, y)$ denotes a point in $\mathbb{R}^{2}$ and $\partial_{x}, \partial_{y}$ respectively denote the partial derivative with respect to the first and to the second coordinate. Given a Lebesgue measurable set $E \subset \mathbb{R}^{2}$, the $\alpha$-perimeter of $E$ is defined as

$$
P_{\alpha}(E)=\sup \left\{\int_{E}\left(\partial_{x} \varphi_{1}+|x|^{\alpha} \partial_{y} \varphi_{2}\right) d x d y: \varphi_{1}, \varphi_{2} \in C_{c}^{1}\left(\mathbb{R}^{2}\right), \max _{\mathbb{R}^{2}}\left(\varphi_{1}^{2}+\varphi_{2}^{2}\right)^{\frac{1}{2}} \leq 1\right\} .
$$

If $\mathcal{P}=P_{\alpha}$, we set $\mathscr{P}_{\alpha}=\mathscr{P}_{P_{\alpha}}$, see definition (1.1). Notice that when $\alpha=0$ the $\alpha$-perimeter is the standard De Giorgi perimeter. If $E \subset \mathbb{R}^{2}$ is a bounded set with Lipschitz boundary, we have

$$
P_{\alpha}(E)=\int_{\partial E} \sqrt{\left(N_{x}^{E}(x, y)\right)^{2}+|x|^{2 \alpha}\left(N_{y}^{E}(x, y)\right)^{2}} d \mathcal{H}^{1}(x, y)
$$

where $N^{E}=\left(N_{x}^{E}, N_{y}^{E}\right)$ is the outer unit normal to $E$ and $\mathcal{H}^{1}$ is the one dimensional Hausdorff measure, see [14, Proposition 2.1]. By the representation formula (1.7), it is clear that $x$ translations modify the $\alpha$-perimeter with $\alpha>0$, i.e., there exists $E \subset \mathbb{R}^{2}$ such that $P_{\alpha}(E)=$ $P_{\alpha}(E+(\bar{x}, 0))$, if and only if $|\bar{x}|=0$. This is an essential difference with the classical perimeter, or with the one defined in Carnot groups.

An important feature of the Grushin plane is that isoperimetric sets are completely characterized. Given $v>0$, the isoperimetric problem for the $\alpha$-perimeter is

$$
\min \left\{P_{\alpha}(E): E \subset \mathbb{R}^{2}, \mathcal{L}^{2}(E)=v\right\},
$$

where $\mathcal{L}^{2}$ denotes the two-dimensional Lebesgue measure. Solutions to 1.8 have been studied in [22], and in [14] in Grushin structures of dimension $n \geq 2$ : up to a vertical translation $\tau_{t}(x, y)=(x, y+t)$ and an anisotropic dilation $\delta_{\lambda}(x, y)=\left(\lambda x, \lambda^{\alpha+1} y\right)$, the unique solution to problem (1.8), called isoperimetric set, is

$$
E_{\text {isop }}^{\alpha}=\left\{(x, y) \in \mathbb{R}^{2}:|y|<\varphi_{\alpha}(|x|),|x|<1\right\}, \quad \varphi_{\alpha}(r)=\int_{\arcsin r}^{\frac{\pi}{2}}(\sin t)^{\alpha+1} d t, r>0 .
$$

The one parameter family of dilations $\delta_{\lambda}$ is such that $P_{\alpha}\left(\delta_{\lambda} E\right)=\lambda^{Q-1} P_{\alpha}(E), \mathcal{L}^{2}\left(\delta_{\lambda} E\right)=$ $\lambda^{Q} \mathcal{L}^{2}(E)$, where $Q=\alpha+2$ is called homogeneous dimension. In particular, (1.9) implies the validity of the following sharp isoperimetric inequality for any measurable set $E \subset \mathbb{R}^{2}$ with finite measure:

$$
\mathcal{L}^{2}(E) \leq c(\alpha) P_{\alpha}(E)^{\frac{Q}{Q-1}}, \quad c(\alpha)=\frac{\alpha+1}{\alpha+2}\left(2 \int_{0}^{\pi} \sin ^{\alpha}(t) d t\right)^{-\frac{1}{\alpha+1}} .
$$

When $\alpha=1$, the profile function $\varphi_{\alpha}$ corresponds to the conjectured isoperimetric profile function of the Heisenberg groups, see [21, 23, 25, 28, 29, 30]. 
Quantitative isoperimetric inequalities have been studied in Riemannian manifolds providing results in the Gauss space (see [5]), in the $n$-Sphere (see [2]) and in the Hyperbolic space (see [3]). Quantitative isoperimetric inequalities in a sub-Riemannian setting are presented in [15] in the case of the Heisenberg group. An interesting task would be to study quantitative isoperimetric inequalities in spaces with less isometries, such as the Grushin plane. However, in this paper we will show some unexpected obstacles that prevent an adaptation of the techniques in [16] to the Grushin plane. In particular we will show that, when $\alpha>0$, solutions to the minimal partition problem (1.4) are not obtained in their central part as portions of isoperimetric sets lying in a vertical stripe.

From now on we consider $\mathcal{P}=P_{\alpha}$ and $\mathscr{P}_{\mathcal{P}}=\mathscr{P}_{\alpha}$. In the first part of this paper we establish existence of solutions.

Theorem 1.1. Let $\alpha \geq 0, v_{1}, v_{2}, h_{1}, h_{2} \geq 0$. There exists a solution $E=E^{l} \cup E^{c} \cup E^{r} \in$ $\mathcal{A}\left(v_{1}, v_{2}, h_{1}, h_{2}\right)$ to the minimal partition problem with trace constraint (1.4) such that $E^{c}$ is a convex set and $E^{l}, E^{r}$ have Lipschitz boundaries.

Minimizers as in Theorem 1.1 are called regular. In Proposition 3.2 we show, under a technical assumption, that any regular minimizer $E \in \mathcal{A}$ assumes the least possible traces at the partitioning point $x_{0}>0$, i.e., $\operatorname{tr}_{x_{0}-}^{x} E=\left[-h_{1}, h_{1}\right]$, and $\operatorname{tr}_{x_{0}+}^{x} E=\left[-h_{2}, h_{2}\right]$.

When $\alpha \in\{0,1\}$ and $v_{2}=h_{2}=0$, the geometry of regular solutions can be described in a more precise way.

Theorem 1.2. Let $\alpha \in\{0,1\}$. Given $v_{1}, h_{1}>0$, let $E=E^{l} \cup E^{c} \cup E^{r} \in \mathcal{A}\left(v_{1}, 0, h_{1}, 0\right)$ be a regular solution for Problem (1.4) such that $\operatorname{tr}_{x_{0}}^{-} E=\left[-h_{1}, h_{1}\right]$. Then $E^{c}=\left\{(x, y) \in \mathbb{R}^{2}:|y|<\right.$ $\left.f(x),|x|<x_{0}\right\}$, where the function $f$ is given by

$$
f(r)=\lambda^{\alpha+1} \varphi_{\alpha}\left(\frac{r}{\lambda}\right)+y,
$$

for some $\lambda=\lambda\left(\alpha, v_{1}, h_{1}\right)>0$ and $y=y\left(\alpha, v_{1}, h_{1}\right) \leq 0$ such that $y=0$ if and only if $\alpha=0$.

Due to the presence of the vertical translation $y$ in $(1.10)$, we deduce by Theorem 1.2 that a regular solution of the minimal partition problem is not obtained as the portion of an isoperimetric set $\delta_{\lambda}\left(E_{\text {isop }}^{\alpha}\right)$ lying in a vertical stripe, unless $\alpha=0$. This result shows a delicate point where the techniques of [16] fail in the case of the Grushin geometry.

The paper is organized as follows. In Section 2, we prove existence of regular solutions of the minimal partition problem. The argument is divided into several steps. Lemma 2.1 is an approximation theorem, that generalizes the classical results in [12. In Lemma 2.2 we show how to modify a set in the class $\mathcal{A}$ in order to decrease the perimeter $\mathscr{P}_{\alpha}$ and gain some regularity properties. Finally, in Theorem 2.4 we combine lower semicontinuity of the $\alpha$-perimeter together with a compactness theorem for sets of finite $\alpha$-perimeter to prove existence of minimizers.

In Section 3, we characterize regular solutions of the minimal partition problem. We call the profile function of a set $E \in \mathscr{S}_{x} \cap \mathscr{S}_{y}^{*}$, the measurable function $f:[0, \infty) \rightarrow[0, \infty)$ such that $E=\left\{(x, y) \in \mathbb{R}^{2}:|y|<f(|x|)\right\}$. In Propostion 3.1 we find differential equations for the profile functionof a regular minimizer. In Section 3.2 we prove, under a technical assumption, that regular solutions of the minimal partition problem (1.4) satisfy the trace equality (1.3). We conclude showing formula (1.10) in Proposition 3.5, which is proved under the assumptions $\alpha \in\{0,1\}, v_{1}, h_{1}>0$ and $v_{2}=h_{2}=0$. To this purpose, we use the differential equations of Proposition 3.1 to write the parameters $\lambda$ and $y$ in terms of $\alpha$ and of the given constraints.

Appendix $\mathrm{A}$ is dedicated to the notion of trace of a $y$-Schwarz symmetric set. 


\section{EXISTENCE OF MINIMIZERS}

In this section we prove existence of solutions to problem (1.4). The proof is divided into several steps that we present in Lemmas 2.1 and 2.2 .

We first introduce some notation. For any set $E \subset \mathbb{R}^{2}$ and $t>0$ we let

$$
E_{t-}^{x}=\{(x, y) \in E:|x|<t\} \quad \text { and } \quad E_{t}^{x}=\{(x, y) \in E:|x|=t\}
$$

In the following, we use the short notation $\{|x|<t\}$ for $\left\{(x, y) \in \mathbb{R}^{2}:|x|<t\right\}$.

The next lemma is a refinement of the approximation theorem by smooth sets for sets with finite $\alpha$-perimeter, see [12, Theorem 2.2.2]. We say that a set $E \subset \mathbb{R}^{2}$ is locally Lipschitz (resp. locally $C^{\infty}$ ) if its boundary $\partial E$ is a locally Lipschitz (resp. locally $C^{\infty}$ ) curve.

Lemma 2.1 (Approximation by smooth sets). Given $v_{1}, v_{2}, h_{1}, h_{2}>0$, let $E \in \mathcal{A}\left(v_{1}, v_{2}, h_{1}, h_{2}\right)$ be a set with finite $\alpha$-perimeter. Let $x_{0}>0$ be the partitioning point for $E$ and $y_{0}^{ \pm}$be such that $\operatorname{tr}_{x_{0} \pm}^{x} E=\left[-y_{0}^{ \pm}, y_{0}^{ \pm}\right]$. Then there exists a sequence of locally $C^{\infty}$ sets $\mathcal{E}_{j} \in \mathscr{S}_{x} \cap \mathscr{S}_{y}^{*}, j \in \mathbb{N}$ such that

- $\lim _{j \rightarrow \infty} P_{\alpha}\left(\left(\mathcal{E}_{j}\right)_{x_{0}-}^{x}\right)=P_{\alpha}\left(E_{x_{0}-}^{x}\right)$ and $\lim _{j \rightarrow \infty} P_{\alpha}\left(\mathcal{E}_{j} \backslash\left(\mathcal{E}_{j}\right)_{x_{0}-}^{x}\right)=P_{\alpha}\left(E \backslash E_{x_{0}-}^{x}\right)$;

- $\lim _{j \rightarrow \infty} \mathcal{L}^{2}\left(\left(\mathcal{E}_{j}\right)_{x_{0}-}^{x}\right)=\mathcal{L}^{2}\left(E_{x_{0}-}^{x}\right)$ and $\lim _{j \rightarrow \infty} \mathcal{L}^{2}\left(\mathcal{E}_{j} \backslash\left(\mathcal{E}_{j}\right)_{x_{0}-}^{x}\right)=\mathcal{L}^{2}\left(E \backslash E_{x_{0}-}^{x}\right)$;

- if $\operatorname{tr}_{x_{0} \pm}^{x} \mathcal{E}_{j}=\left[-q_{j}^{ \pm}, q_{j}^{ \pm}\right]$, for some $q_{j}^{ \pm} \geq 0$, we have $q_{j}^{ \pm} \rightarrow y_{0}^{ \pm}$as $j \rightarrow \infty$.

Proof. Let $J \in C_{c}^{\infty}(B(0,1))$, be a positive symmetric mollifier and, for $\varepsilon>0$, let $J_{\varepsilon}(p)=$ $\frac{1}{\varepsilon^{2}} J(|p| / \varepsilon), p \in \mathbb{R}^{2}$. Following [17, Theorem 1.24], we can choose $t \in(0,1)$ such that, given any sequence $\varepsilon_{j} \rightarrow 0^{+}, j \rightarrow \infty$ the sets $\mathcal{E}_{j}=\left\{p \in \mathbb{R}^{2}: J_{\varepsilon_{j}} * \chi_{E}(p)>t\right\}$ are locally smooth sets satisfying 2.1b), and

$$
\left.\lim _{j \rightarrow \infty} P_{\alpha}\left(\mathcal{E}_{j} ;\left(\mathcal{E}_{j}\right)_{x_{0}-}^{x}\right)=P_{\alpha}\left(E ; E_{x_{0}-}^{x}\right), \lim _{j \rightarrow \infty} P_{\alpha}\left(\mathcal{E}_{j} ; \mathcal{E}_{j} \backslash\left(\mathcal{E}_{j}\right)_{x_{0}-}^{x}\right\}\right)=P_{\alpha}\left(E ; E \backslash E_{x_{0}-}^{x}\right) .
$$

By symmetry of $J$, the sets $\mathcal{E}_{j}$ are $x$-symmetric. We show that they are also $y$-Schwarz symmetric. In fact, since $E$ is $y$-Schwarz symmetric, we have $\chi_{E}\left(\bar{x}-x^{\prime}, \bar{y}-y^{\prime}\right) \leq \chi_{E}\left(\bar{x}-x^{\prime}, y-y^{\prime}\right)$ for $\left(x^{\prime}, y^{\prime}\right) \in \mathbb{R}^{2}$ and $|y|<|\bar{y}|$. Hence, setting $h_{\varepsilon_{j}}=J_{\varepsilon} * \chi_{E}$, we obtain

$$
\begin{aligned}
t<h_{\varepsilon_{j}}(\bar{x}, \bar{y})=\int_{B_{\varepsilon_{j}}(0)} J_{\varepsilon}\left(x^{\prime}, y^{\prime}\right) \chi_{E}\left(\bar{x}-x^{\prime}, \bar{y}-y^{\prime}\right) d x^{\prime} d y^{\prime} \\
\quad \leq \int_{B_{\varepsilon_{j}}(0)} J_{\varepsilon}\left(x^{\prime}, y^{\prime}\right) \chi_{E}\left(\bar{x}-x^{\prime}, y-y^{\prime}\right) d x^{\prime} d y^{\prime}=h_{\varepsilon_{j}}(\bar{x}, y),
\end{aligned}
$$

which implies $(\bar{x}, y) \in \mathcal{E}_{j}$ for $|y|<|\bar{y}|$.

We prove claim 2.1c). Let $\phi_{j}$ denote the profile function of $\mathcal{E}_{j}$. Then we have $\operatorname{tr}_{x_{0} \pm}^{x} \mathcal{E}_{j}=$ $\left[-q_{j}^{ \pm}, q_{j}^{ \pm}\right]$, where (see Remark A.3

$$
q_{j}^{-}=\lim _{x \rightarrow x_{0}^{-}} \phi_{j}\left(x_{0}\right), \quad q_{j}^{+}=\lim _{x \rightarrow x_{0}^{+}} \phi_{j}\left(x_{0}\right) .
$$

In the same way, for $0<\sigma<y_{0}^{+}$, there exists $\delta=\delta(\sigma)>0$ such that

$$
\left|f(x)-y_{0}^{+}\right|<\sigma \quad \text { for } \quad x_{0}<x<x_{0}+\delta,
$$

where $f$ is the profile function of $E$. We first claim that there exists $\bar{j}=\bar{j}(\sigma)$ such that

$$
(x-\xi, y-\eta) \in E \quad \text { for } \quad(\xi, \eta) \in B\left(0, \varepsilon_{j}\right)
$$


for any $j \geq \bar{j}$ and for $(x, y)$ sufficiently far from $\left(x_{0}, y_{0}^{+}\right)$. To prove it, choose $\bar{j}(\sigma) \in \mathbb{N}$ to have $\varepsilon_{\bar{j}}<\min \{\sigma, \delta(\sigma) / 4\}$ and let $(x, y) \in A_{\sigma}=\left(x_{0}+\varepsilon_{j}, x_{0}+\frac{\delta}{2}\right) \times\left(0, y_{0}^{+}-2 \sigma\right)$ for $j \geq \bar{j}$. Then, for $-\varepsilon_{j}<\xi, \eta<\varepsilon_{j}$, we get $x-\xi \in\left(x_{0}, x_{0}+\delta\right)$. Hence 2.3) leads to $y-\eta<f(x-\xi)$ and the claim is proved.

We deduce from (2.4) that $A_{\sigma} \subset \mathcal{E}_{j}$ for $j \geq j(\sigma)$. In fact, if $(x, y) \in A_{\sigma}$ we have

$$
h_{\varepsilon_{j}}(x, y)=\int_{B\left(0, \varepsilon_{j}\right)} J_{\varepsilon_{j}}(\xi, \eta) \chi_{E}(x-\xi, y-\eta) d \xi d \eta=\int_{B\left(0, \varepsilon_{j}\right)} J_{\varepsilon_{j}}(\xi, \eta) d \xi d \eta=1>t .
$$

In particular,

$$
\left(-y_{0}^{+}+2 \sigma, y_{0}^{+}-2 \sigma\right) \subset \operatorname{tr}_{\left(x_{0}+\varepsilon_{j}\right)+}^{x} \mathcal{E}_{j} \quad \text { for every } j>\bar{j}(\sigma) .
$$

Similarly, we can choose $\overline{\bar{j}}(\sigma) \in \mathbb{N}$ such that

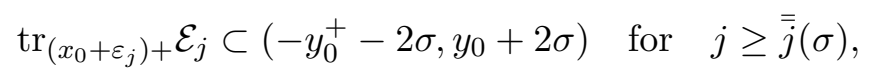

and $(2.1 \mathrm{c})$ for the right trace is proved. The argument to prove $2.1 \mathrm{c}$ ) for the left trace is analogous. Statement 2.1a follows from 2.1c and 2.2.

Lemma 2.2 (Regularization). Let $v_{1}, v_{2}, h_{1}, h_{2} \geq 0$ and $E \in \mathcal{A}\left(v_{1}, v_{2}, h_{1}, h_{2}\right)$ be a locally $C^{\infty}$ set with finite $\alpha$-perimeter. Then, there exists a set $\tilde{E} \in \mathcal{A}\left(v_{1}, v_{2}, h_{1}, h_{2}\right)$ such that, if $\tilde{x}_{0}$ is the partitioning point for $\tilde{E}$, there holds:

(1) $\tilde{E}_{\tilde{x}_{0}-}^{x}$ is convex and $\tilde{E} \backslash \tilde{E}_{\tilde{x}_{0}-}^{x}$ has locally Lipschitz boundary;

(2) $\mathscr{P}_{\alpha}(\tilde{E}) \leq \mathscr{P}_{\alpha}(E)$, in particular $P_{\alpha}\left(\tilde{E}_{\tilde{x}_{0}-}^{x}\right) \leq P_{\alpha}\left(E_{x_{0}-}^{x}\right)$ and $P_{\alpha}\left(\tilde{E} \backslash \tilde{E}_{\tilde{x}_{0}-}^{x}\right) \leq P_{\alpha}\left(E \backslash E_{x_{0}-}^{x}\right)$ where $x_{0}$ is the partitioning point for $E$.

Proof. Let $\operatorname{tr}_{x_{0}-}^{x} E=\left[-q^{-}, q^{-}\right]$and $\operatorname{tr}_{x_{0}+}^{x} E=\left[-q^{+}, q^{+}\right]$. We divide the proof into the following steps, corresponding to operations performed on the set $E$.

Step 1. (Gluing around the y-axis). Starting from $E$, we construct a set $\hat{E} \in \mathscr{S}_{x} \cap \mathscr{S}_{y}^{*}$ such that there exist $0<\hat{x}_{0} \leq x_{0}$ satisfying:

(1) the Euclidean outer unit normal to $\hat{E}$ exists outside a set of $\mathcal{H}^{1}$-measure zero;

(2) if $\hat{\phi}:[0, \infty) \rightarrow[0, \infty)$ denotes the profile function of $\hat{E}$ and $\hat{D}=\inf \{d \geq 0: \hat{\phi}(x)=$ 0 for $x \geq d\}$, then $\mathcal{H}^{1}(\{x \in[0, \hat{D}]: \hat{\phi}(x)=0\})=0$;

(3) $P_{\alpha}\left(\hat{E}_{\hat{x}_{0}-}^{x}\right) \leq P_{\alpha}\left(E_{x_{0}-}^{x}\right)$ and $P_{\alpha}\left(\hat{E} \backslash \hat{E}_{\hat{x}_{0}-}^{x}\right) \leq P_{\alpha}\left(E \backslash E_{x_{0}-}^{x}\right)$;

(4) $\mathcal{L}^{2}\left(\hat{E}_{\hat{x}_{0}-}^{x}\right)=\mathcal{L}^{2}\left(E_{x_{0}-}^{x}\right)$ and $\mathcal{L}^{2}\left(\hat{E} \backslash \hat{E}_{\hat{x}_{0}-}^{x}\right)=\mathcal{L}^{2}\left(E \backslash E_{x_{0}-}^{x}\right)$;

(5) $\operatorname{tr}_{\hat{x}_{0}-}^{x} \hat{E}=\operatorname{tr}_{x_{0}}^{-} E$ and $\operatorname{tr}_{\hat{x}_{0}+}^{x} \hat{E}=\operatorname{tr}_{x_{0}}^{+} E$.

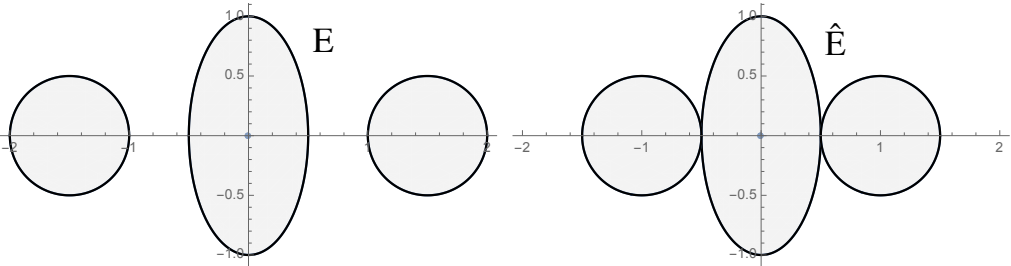

Figure 3. From $E$ to $\hat{E}$.

Let $\phi:[0, \infty) \rightarrow[0, \infty)$ be the profile function of $E$. Let $D=\inf \{d \geq 0: \phi(x)=0$ for $x \geq d\}$. Define the set $Z:=\{x \in[0, D]: \phi(x)=0\}$ and write $Z=Z^{1} \cup Z^{2}$ with

$$
\begin{aligned}
& Z^{1}=\{x \in[0, D]: \phi(x)=0 \text { and } \phi(\xi) \neq 0 \text { for } \xi \in(x-\delta, x+\delta) \backslash\{x\} \text { for some } \delta>0\}, \\
& Z^{2}=\{x \in[0, D]: \exists \delta>0: \phi(\xi)=0 \text { for } \xi \in(x-\delta, x] \text { or } \xi \in[x, x+\delta)\} .
\end{aligned}
$$


By symmetry and smoothness of $E$, we have $Z^{1}=\emptyset$. On the other hand, the set $Z^{2}$ is the union of at most countably many open intervals, being the complement in $\mathbb{R}$ of the closed set $\{x \in \mathbb{R}:(x, 0) \in \bar{E}\}$. Let

$$
Z^{2}=\bigcup_{k \in \mathfrak{I}}\left(a_{k}, b_{k}\right) \cup \bigcup_{k \in \mathfrak{J}}\left(c_{k}, d_{k}\right) \cup \bigcup_{k \in \mathfrak{I}}\left(-b_{k},-a_{k}\right) \cup \bigcup_{k \in \mathfrak{J}}\left(-d_{k},-c_{k}\right),
$$

for $0 \leq a_{1}<b_{1}<a_{2}<b_{2}<\cdots \leq x_{0}<c_{1}<d_{1}<c_{2}<d_{2}<\cdots \leq D$ and $\mathfrak{I}, \mathfrak{J} \subset \mathbb{N}$. We rearrange $E$ in at most countably many steps, each one corresponding to an interval $\left(a_{k}, b_{k}\right)$.

Associated with the interval $\left(a_{1}, b_{1}\right)$, define the set

$$
E_{1}=(E)_{a_{1}-}^{x} \cup\left\{\left(x+a_{1}-b_{1}, y\right):(x, y) \in E, x>b_{1}\right\} \cup\left\{\left(x+b_{1}-a_{1}, y\right):(x, y) \in E, x<-b_{1}\right\}
$$

obtained by "filling the hole" $\left(a_{1}, b_{1}\right) . E$ is $x$-symmetric and $y$-Schwarz symmetric. Moreover, letting $x_{1}=x_{0}+a_{1}-b_{1}<x_{0}$, we have

$$
\mathcal{L}^{2}\left(\left(E_{1}\right)_{x_{1}-}^{x}\right)=\mathcal{L}^{2}\left(E_{x_{0}-}^{x}\right), \quad \mathcal{L}^{2}\left(E_{1} \backslash\left(E_{1}\right)_{x_{1}-}^{x}\right)=\mathcal{L}^{2}\left(E \backslash E_{x_{0}-}^{x}\right)
$$

and $\operatorname{tr}_{x_{1} \pm}^{x} E_{1}=\operatorname{tr}_{x_{0} \pm}^{x} E$. We show that $P_{\alpha}\left(\left(E_{1}\right)_{x_{1}-}^{x}\right) \leq P_{\alpha}\left(E_{x_{0}-}^{x}\right)$. Let $N_{1}(p)=\left(N_{1 x}(p), N_{1 y}(p)\right)$ be the Euclidean outer unit normal to $E_{1}$ at $p=(x, y) \in \mathbb{R}^{2}$, for $|x| \neq a_{1}$. If $N=\left(N_{x}, N_{y}\right)$ is the Euclidean outer unit normal to $\partial E$, we have

$$
N_{1}(x, y)=N\left(x-a_{1}+b_{1}, y\right) \text { if } x>a_{1} \text { and } N_{1}(x, y)=N\left(x-b_{1}+a_{1}, y\right) \text { if } x<-a_{1} .
$$

The claim then follows by the representation formula (1.7), observing that, given $x \in \partial\left(\left(E_{1}\right)_{x_{1}-}^{x} \backslash\right.$ $\left.\left(E_{1}\right)_{a_{1}-}^{x}\right)$ we have

$$
|x|^{2 \alpha} N_{y}\left(x-a_{1}+b_{1}, y\right)^{2}=\left|\bar{x}-b_{1}+a_{1}\right|^{2 \alpha} N_{y}(\bar{x}, y)^{2} \leq|\bar{x}|^{2 \alpha} N_{y}(\bar{x}, y)^{2},
$$

for $\bar{x}=x-a_{1}+b_{1} \in \partial\left(E_{x_{0}}^{x} \backslash E_{b_{1}}^{x}\right)$.

We repeat this procedure for every interval $\left(a_{k}, b_{k}\right), k \in \mathfrak{I}$. After at most countably many operations, we obtain a $x$-symmetric and $y$-Schwarz symmetric set $\hat{E}$ satisfying claims 3,4 and 5 for $\hat{x}_{0}=x_{0}-\sum_{i \in \mathcal{I}}\left(b_{i}-a_{i}\right)$. Repeating this argument for the intervals $\left(c_{k}, d_{k}\right), k \in \mathfrak{J}$, we define the set $\hat{E}$ that satisfies also claims 1 and 2 . In particular, letting

$$
\hat{Z}=\left\{a_{k}-\sum_{i=1}^{k-1}\left(b_{i}-a_{i}\right): k \in \mathfrak{I}\right\} \cup\left\{c_{k}-\sum_{i=1}^{k-1}\left(d_{i}-c_{i}\right): k \in \mathfrak{J}\right\},
$$

the outer unit normal to $\hat{E}$ exists outside the set $\left\{(x, y) \in \mathbb{R}^{2}:|x| \in \hat{Z}\right\}$.

Step 2. (Reflection in the vertical direction) We rearrange the set $\hat{E}$ into a $x$-symmetric and $y$-Schwarz symmetric set $\hat{\hat{E}}$ with profile function $\hat{\hat{\phi}}:[0, \infty) \rightarrow[0, \infty)$ such that

(1) The Euclidean outer unit normal to $\hat{\hat{E}}$ exists outside a set of $\mathcal{H}^{1}$-measure zero;

(2) $\hat{\hat{\phi}}(|x|) \geq q^{-}$for $x \in \mathbb{R},|x|<\hat{x}_{0}$;

(3) $P_{\alpha}\left((\hat{\hat{E}})_{\hat{x}_{0}-}^{x}\right) \leq P_{\alpha}\left(\hat{E}_{\hat{x}_{0}-}^{x}\right)$ and $P_{\alpha}\left(\hat{\hat{E}} \backslash(\hat{\hat{E}})_{\hat{x}_{0}-}^{x}\right)=P_{\alpha}\left(\hat{E} \backslash(\hat{E})_{\hat{x}_{0}-}^{x}\right)$;

(4) $\mathcal{L}^{2}\left((\hat{\hat{E}})_{\hat{x}_{0}-}^{x}\right) \geq \mathcal{L}^{2}\left(\hat{E}_{\hat{x}_{0}-}^{x}\right)$ and $\mathcal{L}^{2}\left(\hat{\hat{E}} \backslash(\hat{\hat{E}})_{\hat{x}_{0}-}^{x}\right)=\mathcal{L}^{2}\left(\hat{E} \backslash(\hat{E})_{\hat{x}_{0}-}^{x}\right)$;

(5) $\operatorname{tr}_{\hat{x}_{0}-}^{x} \hat{\hat{E}}=\operatorname{tr}_{\hat{x}_{0}-}^{x} \hat{E}$ and $\operatorname{tr}_{\hat{x}_{0}+}^{x} \hat{\hat{E}}=\operatorname{tr}_{\hat{x}_{0}+}^{x} \hat{E}$.

We define the rearranged function $\hat{\hat{\phi}}:[0, \infty) \rightarrow[0, \infty)$,

$$
\hat{\hat{\phi}}(x)= \begin{cases}\left|\hat{\phi}(x)-q^{-}\right|+q^{-} & \text {if }|x|<\hat{x}_{0} \\ \hat{\phi}(x) & \text { if }|x|>\hat{x}_{0}\end{cases}
$$


Let $\hat{\hat{E}}$ be the $x$ - and $y$-symmetric set generated by $\hat{\hat{\phi}}$ (see Figure 4 ).
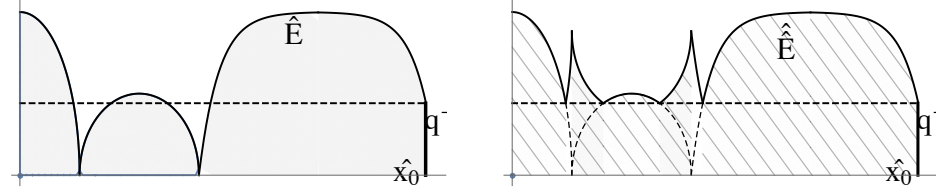

Figure 4 . The set $\hat{E}$ and the rearranged $\hat{\hat{E}}$.

Claims 2, 4 and 5 are obvious. The points where this operation breaks the regularity of the boundary are the ones in $\mathcal{K}=\left\{(x, \hat{\phi}(x)) \in \partial \hat{E}: \hat{\phi}(x)=q^{-}\right.$and $\exists \delta>0: \hat{\phi}(\xi) \neq q^{-}$for $\xi \in$ $(x, x+\delta)$ or $\xi \in(x-\delta, x)\}$. Claim 1 follows since $\mathcal{K}$ is countable.

Moreover, letting $\hat{\hat{N}}=\left(\hat{\hat{N}}_{x}, \hat{\hat{N}}_{y}\right)$ be the outer unit normal to $\hat{\hat{E}}$ outside $\mathcal{K} \cup \hat{Z}$ and $\hat{N}=\left(\hat{N}_{x}, \hat{N}_{y}\right)$ be the outer unit normal to $\partial \hat{E}$, we have for any $(x, y) \in \partial \hat{E} \backslash(\mathcal{K} \cup \hat{Z}),|x|<\hat{x}_{0}$,

$$
\hat{\hat{N}}\left(x,\left|y-q^{-}\right|+q^{-}\right)=\left(\hat{N}_{x}(x, y), \operatorname{sgn}\left(y-q^{-}\right) \hat{N}_{y}(x, y)\right) .
$$

We hence deduce claim 3 by the Representation formula (1.7), by writing

$$
P_{\alpha}\left((\hat{\hat{E}})_{\hat{x}_{0}-}^{x}\right)=\int_{\left\{p \in \partial \hat{E}_{\hat{x}_{0}-}^{x}: \hat{N}_{y}(p) \neq 0\right\}} \sqrt{\hat{N}_{x}^{2}+|x|^{2 \alpha} \hat{N}_{y}^{2}} d \mathcal{H}^{1}+\mathcal{H}^{1}\left(\left\{p \in \partial(\hat{\hat{E}})_{\hat{x}_{0}-}^{x}: \hat{\hat{N}}_{y}(p)=0\right\}\right) .
$$

Step 3. (Convexification and regularization) We finally rearrange the set $\hat{\hat{E}}$ into a $x$-symmetric and $y$-Schwarz symmetric set $\tilde{E}$, such that there exists $0<\tilde{x}_{0}<x_{0}$ satisfying:

(1) $(\tilde{E})_{\tilde{x}_{0}-}^{x}$ is convex, and $\tilde{E} \backslash \tilde{E}_{\tilde{x}_{0}-}^{x}$ is locally lipschitz;

(2) $P_{\alpha}\left((\tilde{E})_{\tilde{x}_{0}-}^{x}\right) \leq P_{\alpha}\left(E_{x_{0}-}^{x}\right)$ and $P_{\alpha}\left(\tilde{E} \backslash(\tilde{E})_{\tilde{x}_{0}-}^{x}\right) \leq P_{\alpha}\left(E \backslash E_{x_{0}-}^{x}\right)$;

(3) $\mathcal{L}^{2}\left((\tilde{E})_{\tilde{x}_{0}-}^{x}\right)=\mathcal{L}^{2}\left(E_{x_{0}-}^{x}\right)$ and $\mathcal{L}^{2}\left(\tilde{E} \backslash(\tilde{E})_{\tilde{x}_{0}-}^{x}\right)=\mathcal{L}^{2}\left(E \backslash E_{x_{0}-}^{x}\right)$;

(4) $\operatorname{tr}_{\tilde{x}_{0}-}^{x} \tilde{E} \supset\left[-q^{-}, q^{-}\right]$and $\operatorname{tr}_{\tilde{x}_{0}+}^{x} \tilde{E} \supset\left[-q^{+}, q^{+}\right]$.

This will conclude the proof.

We introduce the functions

$$
\Psi, \Phi: \mathbb{R}^{2} \rightarrow \mathbb{R}^{2}, \quad \Psi(x, y)=\left(\operatorname{sgn}(x) \frac{|x|^{\alpha+1}}{\alpha+1}, y\right), \quad \Phi(\xi, \eta)=\left(\operatorname{sgn}(\xi)|(\alpha+1) \xi|^{\frac{1}{\alpha+1}}, \eta\right),
$$

which are homeomorphisms such that $\Psi^{-1}=\Phi$. As shown in [22, Prop. 2.3], for any measurable set $F \subset \mathbb{R}^{2}$, we have

$$
P_{\alpha}(F)=P(\Psi(F)) \quad \text { and } \quad \mathcal{L}^{2}(F)=\mu(\Psi(F)),
$$

where $P$ denotes the Euclidean perimeter and $\mu$ is a Borel measure on $\mathbb{R}^{2}$ defined on Borel sets as follows:

$$
\mu(A)=\int_{A}|(\alpha+1) \xi|^{-\frac{\alpha}{\alpha+1}} d \xi d \eta, \quad A \subset \mathbb{R}^{2} \text { Borel. }
$$

Let $F^{\mathrm{c}}=\Psi\left((\hat{\hat{E}})_{\hat{x}_{0}-}^{x}\right) \subset \mathbb{R}^{2}$ and consider its convex envelope in $\mathbb{R}^{2}, \operatorname{co}\left(F^{\mathrm{c}}\right)$. Since $\Phi, \Psi$ preserve the symmetries, the transformed set $\mathcal{E}^{\mathrm{c}}=\Phi\left(\operatorname{co}\left(F^{\mathrm{c}}\right)\right)$ is $x$ - and $y$-Schwarz symmetric. Arguing as in [22, p. 362] we obtain that $\mathcal{E}^{\mathrm{c}}$ is also convex. Moreover, we have

$$
\begin{aligned}
& \mathcal{L}^{2}\left(\mathcal{E}^{\mathrm{c}}\right)=\mu\left(\operatorname{co}\left(F^{\mathrm{c}}\right)\right) \geq \mu\left(F^{\mathrm{c}}\right)=\mathcal{L}^{2}\left((\hat{\hat{E}})_{\hat{x}_{0}-}^{x}\right) \geq \mathcal{L}^{2}\left(\hat{E}_{\hat{x}_{0}-}^{x}\right) \geq \mathcal{L}^{2}\left(E_{x_{0}-}^{x}\right), \\
& P_{\alpha}\left(\mathcal{E}^{\mathrm{c}}\right)=P\left(\operatorname{co}\left(F^{\mathrm{c}}\right)\right) \leq P\left(F^{\mathrm{c}}\right)=P_{\alpha}\left((\hat{\hat{E}})_{\hat{x}_{0}-}^{x}\right), \\
& \operatorname{tr}_{\hat{x}_{0}-}^{x} \mathcal{E}^{\mathrm{c}}=\left[-q^{-}, q^{-}\right] .
\end{aligned}
$$


By (2.5a), we define $\tilde{x}_{0} \in\left[0, \hat{x}_{0}\right]$ such that $\mathcal{L}^{2}\left(\left(\mathcal{E}^{\mathrm{c}}\right)_{\tilde{x}_{0}-}^{x}\right)=\mathcal{L}^{2}\left(E_{x_{0}-}^{x}\right)$. Using a calibration argument as in [14, Proposition 4.2], we deduce that $P_{\alpha}\left(\left(\mathcal{E}^{\mathrm{c}}\right)_{\tilde{x}_{0}-}^{x}\right) \leq P_{\alpha}\left(\mathcal{E}^{\mathrm{c}}\right)$. Moreover, $\left[-q^{-}, q^{-}\right] \subset$ $\operatorname{tr}_{\tilde{x}_{0}-}^{x} \mathcal{E}^{\mathrm{c}}$ since $\mathcal{E}^{\mathrm{c}}$ has a monotone decreasing profile function.

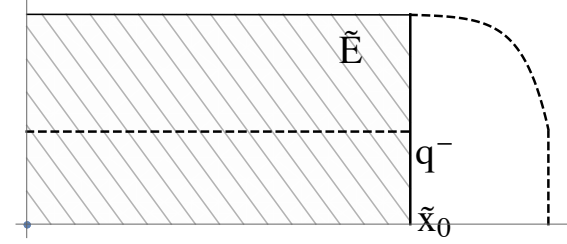

Figure 5. The convexified set $F^{c}$, cut at $\tilde{x}_{0}$.

Define the set $\mathcal{E}=\left(\mathcal{E}^{\mathrm{c}}\right)_{\tilde{x}_{0}-}^{x} \cup\left\{\left(x-\hat{x}_{0}+\tilde{x}_{0}, y\right) \in \mathbb{R}^{2}:(x, y) \in \hat{\hat{E}}, x>\hat{x}_{0}\right\} \cup\left\{\left(x+\hat{x}_{0}-\tilde{x}_{0}, y\right) \in\right.$ $\left.\mathbb{R}^{2}:(x, y) \in \hat{\hat{E}}, x<-\hat{x}_{0}\right\}$. Arguing as in Step 1, we have $P_{\alpha}\left(\mathcal{E} \backslash(\mathcal{E})_{\tilde{x}_{0}-}^{x}\right) \leq P_{\alpha}\left(\hat{\hat{E}} \backslash(\hat{\hat{E}})_{\hat{x}_{0}-}^{x}\right)$, $\mathcal{L}^{2}\left(\mathcal{E} \backslash(\mathcal{E})_{\tilde{x}_{0}-}^{x}\right)=\mathcal{L}^{2}\left(\hat{\hat{E}} \backslash(\hat{\hat{E}})_{\hat{x}_{0}-}^{x}\right)$ and $\operatorname{tr}_{\tilde{x}_{0}+}^{x} \mathcal{E}=\operatorname{tr}_{\hat{x}_{0}+}^{x} \hat{\hat{E}}$

Now, the same argument used to prove 2.5a)-2.5c), shows that the sets $\mathcal{E}^{r}=\Phi(\operatorname{co}(\Psi(\mathcal{E} \cap\{x>$ $\left.\left.\left.\left.\tilde{x}_{0}\right\}\right)\right)\right)$ and $\mathcal{E}^{l}=\left\{(-x, y):(x, y) \in \mathcal{E}^{r}\right\}$ satisfy

$$
\mathcal{L}^{2}\left(\mathcal{E}^{r} \cup \mathcal{E}^{l}\right) \geq \mathcal{L}^{2}\left(\mathcal{E} \backslash(\mathcal{E})_{\tilde{x}_{0}-}^{x}\right), \quad \operatorname{tr}_{\tilde{x}_{0}+} \mathcal{E}^{r}=\operatorname{tr}_{\tilde{x}_{0}+} \mathcal{E}, \quad P_{\alpha}\left(\mathcal{E}^{l} \cup \mathcal{E}^{r}\right) \leq P_{\alpha}\left(\mathcal{E} \backslash(\mathcal{E})_{\tilde{x}_{0}-}^{x}\right) .
$$

Moreover, since $\operatorname{co}\left(\Psi\left(\mathcal{E} \cap\left\{x>\tilde{x}_{0}\right\}\right)\right)$ is a convex set and $\Phi$ is $C^{\infty}$-smooth outside 0 , the set $\mathcal{E}^{r}$ is locally Lipschitz. Let $r \geq \tilde{x}_{0}$ be such that $\mathcal{L}^{2}\left(\left(\mathcal{E}^{r} \cup \mathcal{E}^{l}\right)_{r-}^{x}\right)=\mathcal{L}^{2}\left(E \backslash E_{x_{0}-}^{x}\right)$. The set

$$
\tilde{E}=\left(\mathcal{E}^{c}\right)_{\tilde{x}_{0}-}^{x} \cup\left(\mathcal{E}^{r} \cup \mathcal{E}^{l}\right)_{r-}^{x}
$$

satisfies all the claims of this Step. This concludes the proof.

Remark 2.3. Given a set $E \in \mathcal{A}\left(v_{1}, v_{2}, h_{1}, h_{2}\right)$ with partitioning point $x_{0}>0$ it is always possible to construct a set $\tilde{E} \in \mathcal{A}\left(v_{1}, v_{2}, h_{1}, h_{2}\right)$ such that $\mathscr{P}_{\alpha}(\tilde{E}) \leq \mathscr{P}_{\alpha}(E), \tilde{E}_{x_{0}-}^{x}=E_{x_{0}-}^{x}$, and $\tilde{E} \backslash \tilde{E}_{x_{0}-}^{x}$ is locally Lipschitz. This can be done in the same way as to construct the set $\tilde{E}$ defined in (2.6).

We prove that Problem (1.4) for $P_{\alpha}$ admits regular solutions. Given $v_{1}, v_{2}, h_{1}, h_{2} \geq 0$, let

$$
C_{M P}=\inf \left\{\mathscr{P}_{\alpha}(E): E \in \mathcal{A}\left(v_{1}, v_{2}, h_{1}, h_{2}\right)\right\} .
$$

The constant $C_{M P}$ is positive thanks to the validity of the following isoperimetric inequality: for any $\mathcal{L}^{2}$-measurable set $E \subset \mathbb{R}^{2}$ with finite measure

$$
P_{\alpha}(E) \geq C \mathcal{L}^{2}(E)^{\frac{\alpha+1}{\alpha+2}}
$$

for some geometric constant $C>0$, see [9, 10, 18] (see also [13, Proposition 1.3.4]). In fact, by the formulas

$$
P_{\alpha}\left(E_{t-}^{x}\right)=P_{\alpha}\left(E ; E_{t-}^{x}\right)+\mathcal{H}^{1}\left(E_{t}^{x}\right) \quad \text { and } \quad P_{\alpha}\left(E \backslash E_{t-}^{x}\right)=P_{\alpha}\left(E ; E \backslash E_{t-}^{x}\right)+\mathcal{H}^{1}\left(E_{t}^{x}\right),
$$

holding for a.e. $t>0$ and any set $E$ with finite measure and finite $\alpha$-perimeter (see [14, Proposition 4.1]), we deduce that for $E \in \mathcal{A}, \mathscr{P}_{\alpha}(E) \geq P(E)$. Equality holds if and only if $E \in \mathcal{A}\left(v_{1}, v_{2}, h, h\right)$ is such that $\operatorname{tr}_{x_{0}}^{-} E=\operatorname{tr}_{x_{0}}^{+} E=[-h, h]$, for $h>0$.

Theorem 2.4. Let $v_{1}, v_{2}, h_{1}, h_{2} \geq 0$. There exists a bounded set $E \in \mathcal{A}$ with partitioning point $x_{0} \geq 0$ realizing the infimum in (1.4) for $\mathscr{P}_{\alpha}$ and such that $E_{x_{0}-}^{x}$ is convex, and $E \backslash E_{x_{0}-}^{x}$ is locally Lipschitz. 
Proof. Let $\left(E_{m}\right)_{m \in \mathbb{N}}$ be a minimizing sequence for the infimum in $(1.4)$, namely

$$
E_{m} \in \mathcal{A} \quad \mathscr{P}_{\alpha}\left(E_{m}\right) \leq C_{M P}\left(1+\frac{1}{m}\right) \quad m \in \mathbb{N} .
$$

Let $x_{m}>0$ be the partitioning point for $E_{m}$ and $f_{m}:[0, \infty) \rightarrow[0, \infty)$ be its profile function. Moreover, let $y_{m}^{-}, y_{m}^{+} \geq 0$ be such that $\operatorname{tr}_{x_{m} \pm}^{x} E_{m}=\left[-y_{m}^{ \pm}, y_{m}^{ \pm}\right]$. By Remark A.3.

$$
\lim _{x \rightarrow x_{m}^{+}} f_{m}(x)=y_{m}^{+}, \quad \lim _{x \rightarrow x_{m}^{-}} f_{m}(x)=y_{m}^{-}, \quad \text { with } \quad y_{m}^{+} \geq h_{2}, y_{m}^{-} \geq h_{1} .
$$

Let $m \in \mathbb{N}$. By Lemma 2.1, let $\mathcal{E}_{j}^{m} \in \mathscr{S}_{x} \cap \mathscr{S}_{y}^{*}, j \in \mathbb{N}$ be a sequence of smooth sets satisfying 2.1a)-2.1c for $E=E_{m}$. Define $q_{j m}^{ \pm} \geq 0$ such that $\operatorname{tr}_{x_{m} \pm}^{x} \mathcal{E}_{j}^{m}=\left[-q_{j m}^{ \pm}, q_{j m}^{ \pm}\right]$. For any $j \in \mathbb{N}$, apply Lemma 2.2 to $\mathcal{E}_{j}^{m}$, obtaining a $x$-symmetric and $y$-Schwarz symmetric set $\tilde{\mathcal{E}}_{j}^{m}$, such that there exists $0<\widetilde{x}_{j}^{m}<x_{m}$ satisfying:

(1) $\left(\tilde{\mathcal{E}}_{j}^{m}\right)_{\tilde{x}_{j}^{m}-}^{x}$ is convex, and $\tilde{\mathcal{E}}_{j}^{m} \backslash\left(\tilde{\mathcal{E}}_{j}^{m}\right)_{\tilde{x}_{j}^{m}-}^{x}$ is locally lipschitz;

(2) $P_{\alpha}\left(\left(\tilde{\mathcal{E}}_{j}^{m}\right)_{\tilde{x}_{j}^{m}-}^{x}\right) \leq P_{\alpha}\left(\left(\mathcal{E}_{j}^{m}\right)_{x_{m-}}^{x}\right)$ and $P_{\alpha}\left(\tilde{\mathcal{E}}_{j}^{m} \backslash\left(\tilde{\mathcal{E}}_{j}^{m}\right)_{\tilde{x}_{j}^{m}-}^{x}\right) \leq P_{\alpha}\left(\mathcal{E}_{j} \backslash\left(\mathcal{E}_{j}^{m}\right)_{x_{m}-}^{x}\right)$;

(3) $\mathcal{L}^{2}\left(\left(\tilde{\mathcal{E}}_{j}\right)_{\tilde{x}_{j}-}^{x}\right)=\mathcal{L}^{2}\left(\left(\mathcal{E}_{j}\right)_{x_{0}-}^{x}\right)$ and $\mathcal{L}^{2}\left(\tilde{\mathcal{E}}_{j} \backslash\left(\tilde{\mathcal{E}}_{j}\right)_{\tilde{x}_{j}-}^{x}\right)=\mathcal{L}^{2}\left(\mathcal{E}_{j} \backslash\left(\mathcal{E}_{j}\right)_{x_{0}-}^{x}\right)$;

(4) $\operatorname{tr}_{\tilde{x}_{j}^{m}-}^{x} \tilde{\mathcal{E}}_{j}^{m} \supset\left[-q_{j}^{-}, q_{j}^{-}\right]$and $\operatorname{tr}_{\tilde{x}_{j}+}^{x} \tilde{\mathcal{E}}_{j} \supset\left[-q_{j}^{+}, q_{j}^{+}\right]$.

By (2.1c), for any $m \in \mathbb{N}$ there exists $J(m) \in \mathbb{N}$ such that for $j \geq J(m)$, we have

$$
\left|q_{j m}^{ \pm}-y_{m}^{ \pm}\right| \leq \frac{1}{m}
$$

and

$$
\begin{aligned}
& \left.\mid P_{\alpha}\left(\left(\tilde{\mathcal{E}}_{j}^{m}\right)_{\tilde{x}_{j}^{m}-}^{x}\right)-P_{\alpha}\left(\left(E_{m}\right)_{x_{m}-}^{x}\right)\right)\left|\leq \frac{1}{m}, \quad\right| P_{\alpha}\left(\tilde{\mathcal{E}}_{j}^{m} \backslash\left(\tilde{\mathcal{E}}_{j}^{m}\right)_{\tilde{x}_{j}^{m}-}^{x}\right)-P_{\alpha}\left(E_{m} \backslash\left(E_{m}\right)_{x_{m}-}^{x}\right) \mid \leq \frac{1}{m} \\
& \left.\left.\mid \mathcal{L}^{2}\left(\left(\tilde{\mathcal{E}}_{j}^{m}\right)_{\tilde{x}_{j}^{m}-}^{x}\right)-\mathcal{L}^{2}\left(\left(E_{m}\right)_{x_{m}-}^{x}\right)\right)\left|\leq \frac{1}{m}, \quad\right| \mathcal{L}^{2}\left(\tilde{\mathcal{E}}_{j}^{m} \backslash\left(\tilde{\mathcal{E}}_{j}^{m}\right)_{\tilde{x}_{j}^{m}-}^{x}\right)-\mathcal{L}^{2}\left(E_{m} \backslash\left(E_{m}\right)_{x_{m}-}^{x}\right)\right) \mid \leq \frac{1}{m}
\end{aligned}
$$

Let $\left(j_{m}\right)_{m \in \mathbb{N}}$ be an increasing sequence of integer numbers such that $j_{m} \geq J(m)$ for any $m \in \mathbb{N}$. We choose the diagonal sequence $\tilde{E}_{m}=\tilde{\mathcal{E}}_{j_{m}}^{m}, m \in \mathbb{N}$ and prove that there exists $\ell>0$ such that

$$
\tilde{E}_{m} \subset[-\ell, \ell] \times[-\ell, \ell], \quad \text { for any } \quad m \in \mathbb{N} .
$$

Let $\tilde{x}_{m}=\tilde{x}_{j_{m}}^{m}$. We have

$$
\sup \left\{P_{\alpha}\left(\left(\tilde{E}_{m}\right)_{\tilde{x}_{m-}}^{x}\right): m \in \mathbb{N}\right\}<\infty, \quad \text { and } \sup \left\{P_{\alpha}\left(\tilde{E}_{m} \backslash\left(\tilde{E}_{m}\right)_{\tilde{x}_{m-}}^{x}\right): m \in \mathbb{N}\right\}<\infty,
$$

since $P_{\alpha}\left(\left(\tilde{E}_{m}\right)_{\tilde{x}_{m-}}^{x}\right)+P_{\alpha}\left(\tilde{E}_{m} \backslash\left(\tilde{E}_{m}\right)_{\tilde{x}_{m-}}^{x}\right) \leq 2 C_{M P}+4 h_{1}+4 h_{2}+2$.

We prove that the sequence $\tilde{x}_{m}$ is bounded. Let $\tilde{\phi}_{m}$ be the profile function of $\tilde{E}_{m}$ and assume by contradiction that $x_{m} \rightarrow \infty$ as $m \rightarrow \infty$. In this case, by the representation formula (1.7), we have

$$
P_{\alpha}\left(\left(\tilde{E}_{m}\right)_{\tilde{x}_{m}-}^{x}\right)=\int_{0}^{\tilde{x}_{m}} \sqrt{\tilde{\phi}_{m}(x)^{2}+|x|^{2 \alpha}} d x \geq \int_{0}^{\tilde{x}_{m}}|x|^{\alpha}=\frac{\tilde{x}_{m}^{\alpha+1}}{\alpha+1} \rightarrow \infty, \quad m \rightarrow \infty
$$

which contradicts 2.10 . In the same way, we can see that, if $r_{m}$ is such that $\tilde{E}_{m} \subset\left(\tilde{E}_{m}\right)_{r_{m}-}^{x}$, the sequence $\left(r_{m}\right)_{m \in \mathbb{N}}$ is bounded.

Now, we show that there exists $L \geq 0$ such that $\tilde{E}_{m} \subset\left(\tilde{E}_{m}\right)_{L-}^{y}$. Suppose by contradiction that for any $L \geq 0$, there exists $m=m(L) \in \mathbb{N}$ such that $\left(\tilde{E}_{m}\right)_{\tilde{x}_{m-}}^{x} \backslash\left(\tilde{E}_{m}\right)_{L-}^{y} \neq \emptyset$. By convexity of $\left(\tilde{E}_{m}\right)_{\tilde{x}_{m-}}^{x}$, we can equivalently assume that for any $L \geq 0$ there exists $j(L) \geq 0$ such that

$$
\tilde{\phi}_{m}(0)>L \text { for } m \geq m(L),
$$


We write for $x \in\left(0, \tilde{x}_{m}\right)$

$$
\tilde{\phi}_{m}(x)=-\int_{x}^{\tilde{x}_{m}} \tilde{\phi}_{m}^{\prime}(\xi) d \xi=\int_{x}^{\tilde{x}_{m}}\left|\tilde{\phi}_{m}^{\prime}(\xi)\right| d \xi
$$

then

$$
\tilde{\phi}_{m}(0)=\lim _{x \rightarrow 0} \int_{x}^{\tilde{x}_{m}}\left|\tilde{\phi}_{m}^{\prime}(\xi)\right| d \xi=\int_{0}^{\tilde{x}_{m}}\left|\tilde{\phi}_{m}^{\prime}(\xi)\right| d \xi
$$

which implies, by 2.11

$$
\lim _{m \rightarrow \infty} \int_{0}^{\tilde{x}_{m}}\left|\tilde{\phi}_{m}^{\prime}(\xi)\right| d \xi=\lim _{m \rightarrow \infty} \tilde{\phi}_{m}(0)=\infty
$$

Therefore

$$
P_{\alpha}\left(\left(\tilde{E}_{m}\right)_{\tilde{x}_{m-}}^{x}\right)=4 \int_{0}^{\tilde{x}_{m}} \sqrt{\left(\tilde{\phi}_{m}^{\prime}(x)\right)^{2}+x^{2 \alpha}} d x \geq \int_{0}^{\tilde{x}_{m}}\left|\tilde{\phi}_{m}^{\prime}(x)\right| d x \rightarrow \infty \text { as } m \rightarrow \infty,
$$

which is in contradiction with 2.10$)$. Similarly, we exclude the case that for any $L>0\left(\tilde{E}_{m} \backslash\right.$ $\left.\left(\tilde{E}_{m}\right)_{\tilde{x}_{m}-}^{x}\right) \backslash\left(\tilde{E}_{m}\right)_{L-}^{y} \neq \emptyset$.

Thanks to (2.10), by the compactness theorem for $B V_{\alpha}$ functions (see [18, Thm 1.28 ]), there exists a set $E_{\infty}$ which is the $L_{l o c}^{1}$-limit of $\tilde{E}_{m}$ as $m \rightarrow \infty$. By $(2.9)$, convergence $\chi_{\tilde{E}_{m}} \rightarrow \chi_{E_{\infty}}$ is in $L^{1}\left(\mathbb{R}^{2}\right)$. Moreover, since the sequence $\left(\tilde{x}_{m}\right)_{m \in \mathbb{N}}$ is bounded, we let $x_{\infty} \geq 0$ be the limit up to subsequences of $\tilde{x}_{m}$ as $m \rightarrow \infty$. We have, by (2.8),

$$
\mathcal{L}^{2}\left(\left(E_{\infty}\right)_{\tilde{x}_{\infty}-}^{x}\right)=\lim _{m \rightarrow \infty} \mathcal{L}^{2}\left(\left(\tilde{E}_{m}\right)_{\tilde{x}_{m-}-}^{x}\right)=\lim _{m \rightarrow \infty} \mathcal{L}^{2}\left(\left(E_{m}\right)_{x_{m}-}^{x}\right)=v_{1}
$$

and

$$
\mathcal{L}^{2}\left(E_{\infty} \backslash\left(E_{\infty}\right)_{\tilde{x}_{\infty}-}^{x}\right)=\lim _{m \rightarrow \infty} \mathcal{L}^{2}\left(\tilde{E}_{m} \backslash\left(\tilde{E}_{m}\right)_{\tilde{x}_{m}-}^{x}\right)=\lim _{m \rightarrow \infty} \mathcal{L}^{2}\left(E_{m} \backslash\left(E_{m}\right)_{x_{m}-}^{x}\right)=v_{2}
$$

Now, since $\left(\tilde{E}_{m}\right)_{\tilde{x}_{m-}-}^{x}$, is convex, we can choose a representative for $E_{\infty}$ such that $\left(E_{\infty}\right)_{x_{\infty}-}^{x}$, is convex. By boundedness of the sequence $\tilde{E}_{m}$, let $y_{\infty}^{ \pm} \geq 0$ be such that $\operatorname{tr}_{x_{\infty} \pm}^{x} E_{\infty}=\left[-y_{\infty}^{ \pm}, y_{\infty}^{ \pm}\right]$. Then, by (2.7) and claim 4 at Step 4, we have

$$
y_{\infty}^{-} \geq \lim _{m \rightarrow \infty} \tilde{q}_{m}^{-} \geq \lim _{m \rightarrow \infty} q_{m}^{-} \geq \lim _{m \rightarrow \infty} y_{m}^{-}-\frac{1}{m} \geq h_{1},
$$

equivalently $y_{\infty}^{+} \geq h_{2}$. Hence $E_{\infty} \in \mathcal{A}$.

By the lower semi-continuity of the $\alpha$-perimeter together with $(2.8)$, we have $\mathscr{P}_{\alpha}\left(E_{\infty}\right) \leq$ $\liminf _{m \rightarrow \infty} \mathscr{P}_{\alpha}\left(E_{m}\right)+\frac{2}{m} \leq C_{M P}$. Moreover, by Remark 2.3 applied to $E=E_{\infty}$, there exists a set $\tilde{E}_{\infty} \in \mathcal{A}\left(v_{1}, v_{2}, h_{1}, h_{2}\right)$ such that $\mathscr{P}_{\alpha}\left(\tilde{E}_{\infty}\right) \leq \mathscr{P}_{\alpha}\left(E_{\infty}\right), E_{\infty} \backslash\left(\tilde{E}_{\infty}\right)_{x_{\infty}-}^{x}$ is locally Lipschitz and $\left(\tilde{E}_{\infty}\right)_{x_{\infty}-}^{x}=\left(E_{\infty}\right)_{x_{\infty}-}^{x}$. This concludes the proof.

\section{Profile of Regular solutions}

In this section we describe the solutions to the minimal partition problem (1.4) found in Theorem 2.4. If $E \in \mathcal{A}\left(v_{1}, v_{2}, h_{1}, h_{2}\right)$ with partitioning point $x_{0}>0$ is a bounded solution to Problem (1.4) such that $E_{x_{0}-}^{x}$ is convex and $E \backslash E_{x_{0}-}^{x}$ is locally Lipschitz, we say that $E$ is a regular solution of the minimal partition problem. In this case, writing $E=\left\{(x, y) \in \mathbb{R}^{2}:|y|<f(|x|)\right\}$, the profile function $f:[0, \infty) \rightarrow[0, \infty)$ is decreasing in $\left[0, x_{0}\right)$ and locally Lipschitz in $\left[x_{0}, \infty\right)$. In the following we sometimes consider $f$ to be extended to an even function defined on the whole $\mathbb{R}$, and we still call it the profile function of $E$. 


\subsection{Differential equations for the profile function.}

Proposition 3.1. Let $v_{1}, v_{2}, h_{1}, h_{2} \geq 0$ and $E \subset \mathbb{R}^{2}$ be a regular solution of the minimal partition problem (1.4) for $\mathscr{P}_{\alpha}$ in the class $\mathcal{A}=\mathcal{A}\left(v_{1}, v_{2}, h_{1}, h_{2}\right)$, with partitioning point $x_{0} \geq 0$. Then, writing

the even function $f$ satisfies

$$
E=\left\{(x, y) \in \mathbb{R}^{2}:|y|<f(x)\right\}
$$

$$
\begin{aligned}
& f^{\prime}(x)=-\frac{\operatorname{sgn} x c|x|^{\alpha+1}}{\sqrt{1-c x^{2}}} \quad \text { if } \quad|x|<x_{0}, \\
& f^{\prime}(x)=\frac{(k x+d) x^{\alpha}}{\sqrt{1-(k x+d)^{2}}} \quad \text { if } \quad x>x_{0} . \\
& f^{\prime}(x)=\frac{(k x-d)|x|^{\alpha}}{\sqrt{1-(k x-d)^{2}}} \quad \text { if } \quad x<-x_{0}
\end{aligned}
$$

for some constants $c \geq 0, k, d \in \mathbb{R}$.

Proof. By boundedness of the regular minimizer $E$, let $r_{0}=\inf \left\{r>0: E \subset E_{r-}^{x}\right\}<\infty$.

We prove equation (3.1a). For $\psi_{1} \in C_{c}^{\infty}\left(0, x_{0}\right)$ with $\int \psi_{1}=0$, and $\varepsilon \in \mathbb{R}$, consider the function $x \mapsto f(|x|)+\varepsilon \psi_{1}(|x|), x \in \mathbb{R}$, and define the set

$$
E_{\varepsilon}=\left\{(x, y) \in \mathbb{R}^{2}:|y|<f(|x|)+\varepsilon \psi_{1}(|x|)\right\} \in \mathcal{A} .
$$

By the Representation formula for the $\alpha$-perimeter (1.7), let

$$
p_{1}(\varepsilon)=P_{\alpha}\left(\left(E_{\varepsilon}\right)_{x_{0}-}^{x}\right)=4\left\{\int_{0}^{x_{0}} \sqrt{\left(f^{\prime}+\varepsilon \psi^{\prime}\right)^{2}+|x|^{2 \alpha}} d r+\lim _{x \rightarrow x_{0}^{-}} f(x)\right\} .
$$

By minimality of $E$, we then have

$$
\begin{aligned}
0 & =\left.p_{1}^{\prime}(\varepsilon)\right|_{\varepsilon=0}=\left.4 \int_{0}^{x_{0}} \frac{d}{d \varepsilon}\left(\sqrt{\left(f^{\prime}+\varepsilon \psi_{1}^{\prime}\right)^{2}+x^{2 \alpha}}\right)\right|_{\varepsilon=0} d x \\
& =4 \int_{0}^{x_{0}} \frac{f^{\prime}(x) \psi_{1}^{\prime}(x)}{\sqrt{f^{\prime 2}(x)+x^{2 \alpha}}} d x=-4 \int_{0}^{x_{0}} \frac{d}{d x}\left(\frac{f^{\prime}}{\sqrt{f^{\prime 2}+x^{2 \alpha}}}\right) \psi_{1}(x) d x .
\end{aligned}
$$

By arbitrariness of $\psi_{1}$, we deduce the following second order ordinary differential equation, holding for some $C \in \mathbb{R}$

$$
\frac{d}{d x}\left(\frac{f^{\prime}(x)}{\sqrt{f^{\prime}(x)^{2}+x^{2 \alpha}}}\right)=C \text { for a.e. } 0<x<x_{0} .
$$

The normal form of $(3.2)$ is

$$
f^{\prime \prime}(x)=\frac{\alpha f^{\prime}(x)}{x}+\frac{C}{x^{2 \alpha}}\left(f^{\prime}(x)^{2}+x^{2 \alpha}\right)^{\frac{3}{2}} .
$$

Now, since $E$ is $x$-symmetric, the function $f$ is even, hence $f^{\prime}$ is odd and $f^{\prime \prime}$ is even. This allows us to extend $(3.2)$ to $|x|<x_{0}$. Integrating 3.2 around 0 , we obtain existence of a constant $d \in \mathbb{R}$ such that for some $\delta>0$,

$$
\frac{f^{\prime}(x)}{\sqrt{f^{\prime}(x)^{2}+x^{2 \alpha}}}=C x+d \quad \text { for }|x|<\delta .
$$

Since $f^{\prime}$ is odd we deduce that $d=0$, in fact for $|x|<\delta$

$$
C x+d=\frac{f^{\prime}(x)}{\sqrt{f^{\prime 2}(x)-x^{2 \alpha}}}=-\frac{f^{\prime}(-x)}{\sqrt{f^{\prime 2}(-x)+(-x)^{2} \alpha}}=-(C(-x)+d)=C x-d .
$$


Hence 3.2 reads

$$
\frac{f^{\prime}(x)}{\sqrt{f^{\prime 2}(x)+x^{2 \alpha}}}=C x \text { for }|x|<\delta,
$$

which implies, by monotonicity of $f$, that $C<0$. Letting $c=-C>0$, we hence get the following ordinary differential equation for $f$ :

$$
f^{\prime}(x)=-\operatorname{sgn}(x) \frac{c|x|^{\alpha+1}}{\sqrt{1-c^{2} x^{2}}} \text { for }|x|<\delta .
$$

A solution to the latter equation can be extended up to $(-1 / c, 1 / c)$. This implies $0<x_{0} \leq 1 / c$ and (3.1a) is proved.

To prove (3.1b) and (3.1c), we proceed in the same way, considering a function $\psi_{2} \in C_{c}^{\infty}\left(x_{0}, r_{0}\right)$, with $\int \psi_{2}=0$ and the associated perturbation $f+\eta \psi_{2}$ for $\eta \in \mathbb{R}$. The set $E_{\eta}=\left\{(x, y) \in \mathbb{R}^{2}\right.$ : $\left.|y|<f(|x|)+\varepsilon \psi_{2}(|x|)\right\}$ is inside the class $\mathcal{A}$, hence, as in the previous case, minimality of $E$ leads to

$$
\frac{d}{d x}\left(\frac{f^{\prime}(x)}{\sqrt{f^{\prime}(x)^{2}+x^{2 \alpha}}}\right)=k \quad \text { for } \quad x_{0}<|x|<r_{0}
$$

for some constant $k \in \mathbb{R}$. Let $x_{0}<x<r_{0}$. An integration between $x_{0}$ and $x$ shows that, letting

$$
d=\lim _{x \rightarrow x_{0}^{+}} \frac{f^{\prime}(x)}{\sqrt{f^{\prime}(x)^{2}+x^{2 \alpha}}}-k x_{0},
$$

we have

$$
\frac{f^{\prime}(x)}{\sqrt{f^{\prime}(x)^{2}+x^{2 \alpha}}}=k x+d \quad \text { for } \quad x_{0}<x<r_{0}
$$

which is equivalent to (3.1b). In particular, $|k x+d|<1$ for $x_{0}<x<r_{0}$.

Analogously, for any $x \in\left(-r_{0},-x_{0}\right)$, an integration between $x$ and $-x_{0}$ shows that

$$
\frac{f^{\prime}(x)}{\sqrt{f^{\prime}(x)^{2}+x^{2 \alpha}}}=k x-d \text { for } \quad-r_{0}<x<-x_{0},
$$

which leads to $3.1 \mathrm{c}$ ) and $|k x-d|<1$ for $-r_{0}<x<-x_{0}$.

3.2. Traces of regular solutions. In this section, we study traces of minimizers. What we expect is that if $E \in \mathcal{A}\left(v_{1}, v_{2}, h_{1}, h_{2}\right)$ is a regular solution of the minimal partition problem with partitioning point $x_{0}$, then

$$
\operatorname{tr}_{x_{0}}^{-} E=\left[-h_{1}, h_{1}\right] \text { and } \operatorname{tr}_{x_{0}}^{+} E=\left[-h_{2}, h_{2}\right] .
$$

In Proposition 3.2 we prove the claim for the left trace, under an additional assumption. The case of the right trace is equivalent.

Proposition 3.2. Given $v_{1}, v_{2}, h_{1}, h_{2} \geq 0$, let $E \in \mathcal{A}\left(v_{1}, v_{2}, h_{1}, h_{2}\right)$ be a regular solution of Problem (1.4) for $\mathscr{P}_{\alpha}$ with partitioning point $x_{0}>0$, and let $f:[0, \infty) \rightarrow[0, \infty)$ be its profile function. If

$$
\lim _{x \rightarrow x_{0}^{-}} f^{\prime}(x)>-\infty,
$$

then

$$
\operatorname{tr}_{x_{0}}^{-} E=\left[-h_{1}, h_{1}\right] .
$$




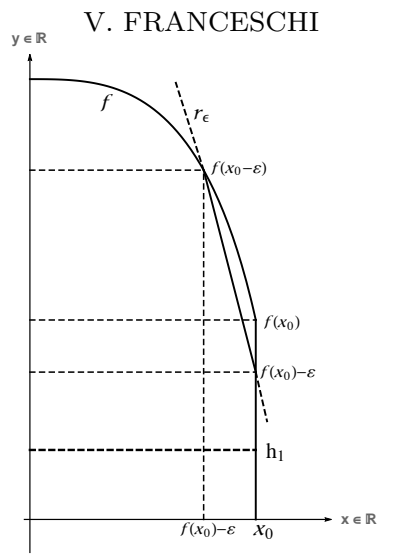

Figure 6. Construction of the set $E_{\varepsilon}$.

Proof. Assume by contradiction that $f\left(x_{0}^{-}\right)>h_{1}$, where $f\left(x_{0}^{-}\right)=\lim _{x \rightarrow x_{0}^{-}} f(x)$. We show that in this case, there exists a set $F \in \mathcal{A}$ such that $P_{\alpha}\left(F_{x_{0}-}^{x}\right)<P_{\alpha}\left(E_{x_{0}-}^{x}\right), P_{\alpha}\left(F \backslash F_{x_{0}-}^{x}\right)=P_{\alpha}\left(E \backslash E_{x_{0}-}^{x}\right)$, hence $\mathscr{P}_{\alpha}(F)<\mathscr{P}_{\alpha}(E)$, which is in contradiction with the minimality of $E$.

For a small parameter $\varepsilon>0$, let $f_{\varepsilon}:\left[0, x_{0}\right) \rightarrow[0, \infty)$ be the function defined by

$$
f_{\varepsilon}(x)= \begin{cases}f(x), & \text { if } 0<x<x_{0}-\varepsilon \\ r_{\varepsilon}(x), & \text { if } x_{0}-\varepsilon<x<x_{0}\end{cases}
$$

where $r_{\varepsilon}$ is the segment connecting the points $\left(x_{0}-\varepsilon, f\left(x_{0}-\varepsilon\right)\right)$ and $\left(x_{0}, f\left(x_{0}^{-}\right)-\varepsilon\right)$, i.e.,

$$
r_{\varepsilon}(x)=m(\varepsilon)\left(x-x_{0}\right)+f\left(x_{0}^{-}\right)-\varepsilon, \quad m(\varepsilon)=\frac{1}{\varepsilon}\left(f\left(x_{0}^{-}\right)-\varepsilon-f\left(x_{0}-\varepsilon\right)\right)<0 .
$$

We define the set $E_{\varepsilon}=\left\{(x, y) \in \mathbb{R}^{2}:|y|<f_{\varepsilon}(|x|)\right\}$ and let $A(\varepsilon)=\left(P_{\alpha}\left(E_{x_{0}-}^{x}\right)-P_{\alpha}\left(E_{\varepsilon}\right)\right) / 4$, $B(\varepsilon)=\left(\mathcal{L}^{2}\left(E_{x_{0}-}^{x}\right)-\mathcal{L}^{2}\left(E_{\varepsilon}\right)\right) / 4$. Let $y_{\varepsilon}=B(\varepsilon) / x_{0}$. We claim that for $\varepsilon>0$ small enough the set

$$
F_{\varepsilon}=\left(E_{\varepsilon}+\left(0, y_{\varepsilon}\right)\right) \cup\left(\left[-x_{0}, x_{0}\right] \times\left[-y_{\varepsilon}, y_{\varepsilon}\right]\right)
$$

obtained by translating $E_{\varepsilon}$ in the vertical direction of the quantity $y_{\varepsilon}$, satisfies

$$
P_{\alpha}\left(F_{\varepsilon}\right)<P_{\alpha}(E)
$$

Since $\mathcal{L}^{2}\left(\left(F_{\varepsilon}\right)_{x_{0}-}^{x}\right)=\mathcal{L}^{2}\left(E_{\varepsilon}\right)+4 x_{0} y_{\varepsilon}=\mathcal{L}^{2}\left(E_{x_{0}-}^{x}\right)$, this contradicts the minimality of $E$ and the statement follows.

We prove (3.4). By invariance under vertical translations of the $\alpha$-perimeter, we have

$$
P_{\alpha}\left(F_{\varepsilon}\right)=P_{\alpha}\left(E_{\varepsilon}\right)+4 y_{\varepsilon}=P_{\alpha}\left(E_{x_{0}-}^{x}\right)-4\left(A(\varepsilon)-\frac{B(\varepsilon)}{x_{0}}\right)
$$

To prove the claim, it is therefore sufficient to show that for $\varepsilon>0$ small enough

$$
x_{0} A(\varepsilon)>B(\varepsilon) .
$$

This follows by a comparison between the Taylor developments of $A$ and $B$. We have

$$
A(\varepsilon)=\left\{\int_{x_{0}-\varepsilon}^{x_{0}} \sqrt{f^{\prime}(x)^{2}+x^{2 \alpha}}-\sqrt{m(\varepsilon)^{2}+x^{2 \alpha}} d x+\varepsilon\right\}, \quad B_{\varepsilon}=\int_{x_{0}-\varepsilon}^{x_{0}} f(x)-r_{\varepsilon}(x) d x .
$$

Hence

$$
\lim _{\varepsilon \rightarrow 0^{+}} A(\varepsilon)=\lim _{\varepsilon \rightarrow 0^{+}} B(\varepsilon)=0 .
$$

Let $f^{\prime}\left(x_{0}^{-}\right)=\lim _{x \rightarrow x_{0}^{-}} f^{\prime}(x)<0$ and $f^{\prime \prime}\left(x_{0}^{-}\right)=\lim _{x \rightarrow x_{0}^{-}} f^{\prime \prime}(x) \leq 0$. We compute

$$
\lim _{\varepsilon \rightarrow 0^{+}} m(\varepsilon)=\lim _{\varepsilon \rightarrow 0^{+}} \frac{f\left(x_{0}^{-}\right)-f\left(x_{0}-\varepsilon\right)}{\varepsilon}-1=f^{\prime}\left(x_{0}^{-}\right)-1 .
$$


Now, by the assumption $f^{\prime}\left(x_{0}^{-}\right)>-\infty$ we also have $f^{\prime \prime}\left(x_{0}^{-}\right)>-\infty$. Hence

$$
\begin{aligned}
m^{\prime}(0) & =\lim _{\varepsilon \rightarrow 0^{+}} m^{\prime}(\varepsilon)=\lim _{\varepsilon \rightarrow 0^{+}} \frac{1}{\varepsilon}\left\{f^{\prime}\left(x_{0}-\varepsilon\right)-\frac{1}{\varepsilon}\left[f\left(x_{0}^{-}\right)-f\left(x_{0}-\varepsilon\right)\right]\right\} \\
& =\lim _{\varepsilon \rightarrow 0^{+}} \frac{1}{\varepsilon}\left\{f^{\prime}\left(x_{0}^{-}\right)-f^{\prime \prime}\left(x_{0}^{-}\right) \varepsilon-\frac{1}{\varepsilon}\left[f\left(x_{0}^{-}\right)-\left(f\left(x_{0}^{-}\right)-f^{\prime}\left(x_{0}^{-}\right) \varepsilon+\frac{f^{\prime \prime}\left(x_{0}^{-}\right)}{2} \varepsilon^{2}\right)\right]+o(\varepsilon)\right\} \\
& =-\frac{f^{\prime \prime}\left(x_{0}^{-}\right)}{2}
\end{aligned}
$$

is positive and finite. Therefore,

$$
\begin{aligned}
A^{\prime}(\varepsilon) & =1+\sqrt{f^{\prime 2}\left(x_{0}-\varepsilon\right)+\left(x_{0}-\varepsilon\right)^{2 \alpha}}-\sqrt{m(\varepsilon)^{2}+\left(x_{0}-\varepsilon\right)^{2 \alpha}}-\int_{x_{0}-\varepsilon}^{x_{0}} \frac{m(\varepsilon) m^{\prime}(\varepsilon)}{\sqrt{m\left(\varepsilon^{2}\right)+x^{2 \alpha}}} d x \\
& \geq \sqrt{f^{\prime 2}\left(x_{0}-\varepsilon\right)+\left(x_{0}-\varepsilon\right)^{2 \alpha}}-\sqrt{m(\varepsilon)^{2}+\left(x_{0}-\varepsilon\right)^{2 \alpha}}
\end{aligned}
$$

that gives

$$
A^{\prime}(0)=\lim _{\varepsilon \rightarrow 0} A^{\prime}(\varepsilon) \geq 1+\sqrt{f^{\prime 2}\left(x_{0}^{-}\right)^{2}+x_{0}^{2 \alpha}}-\sqrt{\left(f^{\prime}\left(x_{0}^{-}\right)-1\right)^{2}+x_{0}^{2 \alpha}}>0 .
$$

The last inequality is justified by the following: for any $c<a<0$, and $b \in \mathbb{R}$,

$$
\sqrt{a_{2}+b_{2}}-\sqrt{c_{2}+b_{2}}>c-a .
$$

We conclude observing that $B^{\prime}(0)=\lim _{\varepsilon \rightarrow 0^{+}} B^{\prime}(\varepsilon)=0$, in fact:

$$
\begin{aligned}
B^{\prime}(\varepsilon) & =f\left(x_{0}-\varepsilon\right)-r_{\varepsilon}\left(x_{0}-\varepsilon\right)+\int_{x_{0}-\varepsilon}^{x_{0}} \frac{d}{d \varepsilon}\left\{f(x)-m(\varepsilon)\left(x-x_{0}\right)-f\left(x_{0}^{-}\right)+\varepsilon\right\} d x \\
& =f\left(x_{0}-\varepsilon\right)+\varepsilon m(\varepsilon)-f\left(x_{0}^{-}\right)+\varepsilon-\int_{x_{0}-\varepsilon}^{x_{0}} 1-m^{\prime}(\varepsilon)\left(x-x_{0}\right) d x \underset{\varepsilon \rightarrow 0}{\longrightarrow} 0
\end{aligned}
$$

Then, (3.5) follows by (3.7) and (3.6).

Remark 3.3. Assume that the profile function of a minimizer as in Theorem 2.4 satisfies

$$
f^{\prime}\left(x_{0}^{-}\right)=\lim _{x \rightarrow x_{0}^{-}} f^{\prime}(x)=-\infty .
$$

Using the notation of Proposition 3.2 , there holds, for $\varepsilon>0$ small enough:

$$
x_{0} A(\varepsilon)-B(\varepsilon)=-\frac{x_{0}^{\alpha+2}}{6 \sqrt{2}}\left(\frac{\varepsilon}{x_{0}}\right)^{\frac{3}{2}}+o\left(\varepsilon^{3 / 2}\right)<0 \quad \text { for } \varepsilon<\varepsilon_{0} .
$$

Hence, the construction proposed in the latter proposition does not apply to this case.

3.3. Center of regular solutions. In this section, we show that we cannot in general expect a regular minimizer for the minimal partition problem to be obtained in its central part as a dilation of the isoperimetric set $E_{\text {isop }}^{\alpha}$. In fact, we show that, for particular choices of $v_{1}, v_{2}, h_{1}, h_{2}>0$ and $\alpha \geq 0$, a regular minimizer $E \in \mathcal{A}$ with partitioning point $x_{0}>0$ does not satisfy for any $\lambda>0$

$$
E_{x_{0}-}^{x}=\left(\delta_{\lambda}^{\alpha} E_{\text {isop }}^{\alpha}\right)_{x_{0}-}^{x} .
$$

Remark 3.4. Let $E$ be a regular solution of the minimal partition problem with partitioning point $x_{0}>0$. Then, by Proposition 3.1, its profile function $f$ is defined on some bounded interval $\left[0, r_{0}\right]$ and it is a locally Lipschitz function satisfying in a weak sense the ordinary differential equations 3.1a)-3.1c . By an elementary argument, that is omitted, it follows that $f \in C^{2}\left(\left[0, x_{0}\right)\right) \cap C^{2}\left(x_{0}, r_{0}\right) \cap C^{2}\left(-r_{0},-x_{0}\right)$. 
Notice that equation (3.1a) is scale invariant, i.e., given $c_{1}, c_{2} \geq 0$ and a solution $g$ to (3.1a) for $c=c_{1}$, the function $g_{\lambda}(x)=\lambda^{\alpha+1} g\left(\frac{x}{\lambda}\right)$, for $\lambda=\frac{c_{1}}{c_{2}}$ is a solution to 3.1a) for $c=c_{2}$. In this sense, in [22, Theorem 3.2], the authors show that the unique solution to equation (3.1a) is the function

$$
\varphi_{\alpha}(x)=\int_{\arcsin |x|}^{\frac{\pi}{2}} \sin ^{\alpha+1}(t) d t, \quad x \in[-1,1]
$$

obtained integrating (3.1a) for $c=1$. In other words, there exist $\lambda>0$ and $y \in \mathbb{R}$ such that

$$
f(x)=\lambda^{\alpha+1} \varphi_{\alpha}\left(\frac{x}{\lambda}\right)+y, \quad|x|<x_{0}
$$

We characterize the parameters $\lambda$ and $y \in \mathbb{R}$ appearing in 3.10 in terms of the data $h_{1}=h$, $v_{1}=v$ in the case of regular solutions of Problem (1.4) with $h_{2}=v_{2}=0$ and $\alpha=0,1$, see Proposition 3.5. We deduce that, if $\alpha=1$, the translation $y$ is strictly negative (see Remark 3.6), hence (3.8) does not hold. On the other hand, in the case when $\alpha=0$, the $\alpha$-perimeter corresponds to the Euclidean perimeter and we prove that regular solutions of the minimal partition problem satisfy (3.8).
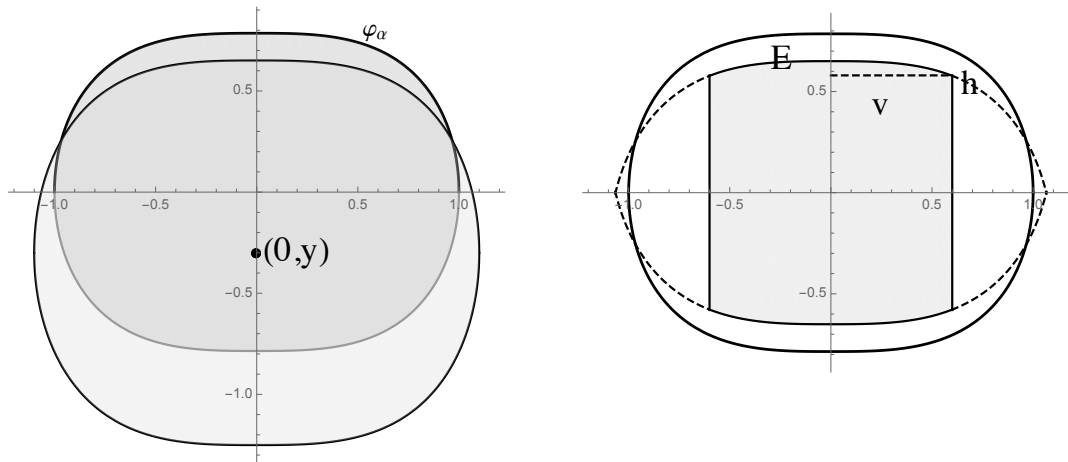

FIgURE 7. For $\alpha>0$, a regular solution for the minimal partition problem is not obtained as a dilation of the isoperimetric set $E_{\text {isop }}^{\alpha}$ in its central part. Its profile function is in fact the profile of an isoperimetric set vertically translated of a negative quantity $y$.

Proposition 3.5. Given $\alpha \in\{0,1\}, h \geq 0$, and $v>0$, let $E \in \mathcal{A}(v, 0, h, 0)$ be a regular solution of Problem (1.4) for $\mathscr{P}_{\alpha}$ with $v_{1}=v, h_{1}=h, v_{2}=h_{2}=0$, and partitioning point $x_{0}>0$, satisfying $\operatorname{tr}_{x_{0}}^{-} E=[-h, h]$. Let $f:\left[0, x_{0}\right] \rightarrow[0, \infty)$ be its profile function. Then there exists $d \in[-1,1]$ such that $f$ solves 3.10 with

$$
\lambda=\frac{x_{0}}{d}, \quad y=h\left\{1-\frac{\varphi_{\alpha}(d)}{d^{\alpha} \sqrt{1-d^{2}}}\right\} .
$$

Proof. By Remark 3.4 there exist $\lambda>0, y \in \mathbb{R}$ such that

$$
f(x)=\lambda^{\alpha+1} \varphi_{\alpha}\left(\frac{x}{\lambda}\right)+y, \quad|x|<x_{0} .
$$

In particular $\lambda=1 / c$. Let $\beta=f(0)>0$, and define

$$
p\left(\beta, c, x_{0}\right)=P_{\alpha}(E)=\int_{0}^{x_{0}} \sqrt{f^{\prime 2}(t)+t^{2 \alpha}} d t=\int_{0}^{x_{0}} \frac{t^{\alpha}}{\sqrt{1-(c t)^{2}}} d t=\frac{1}{c^{\alpha+1}} \int_{0}^{\arcsin c x_{0}} \sin ^{\alpha} \vartheta d \vartheta
$$


Notice that $p$ can be thought of as a functional depending on $\beta, c, x_{0}$ where the triple $\left(\beta, c, x_{0}\right)$ identifies a unique minimizer $E \in \mathcal{A}$ as in the statement. In particular, $p$ is independent of $\beta$, as well as $P_{\alpha}$ is independent of vertical translations. Let $d=c x_{0}$, that leads to $\lambda=x_{0} / d$. With a slight abuse of notation, we write $p$ in terms of $d$ and $x_{0}$ as

$$
p\left(d, x_{0}\right)=x_{0}^{\alpha+1} g_{\alpha}(d), \text { with } g_{\alpha}(d)=\frac{1}{d^{\alpha+1}} \int_{0}^{\arcsin d} \sin ^{\alpha} \vartheta d \vartheta .
$$

We write the volume and trace constraints satisfied by the minimizer $E$ in terms of the parameters $d$ and $x_{0}$. For any $t \in\left(0, x_{0}\right)$, there holds

$$
f(t)=\beta+\int_{0}^{t} f^{\prime}(s) d s=\beta-\frac{x_{0}^{\alpha+1}}{d^{\alpha+1}} \int_{0}^{\arcsin \left(\frac{d}{x_{0}} t\right)} \sin ^{\alpha+1} \vartheta d \vartheta .
$$

Hence the trace constraint $f\left(x_{0}\right)=h$ is equivalent to

$$
\beta=\beta\left(d, x_{0}\right)=h+x_{0}^{\alpha+1} \sigma_{\alpha}(d), \text { with } \sigma_{\alpha}(d)=\frac{1}{d^{\alpha+1}} \int_{0}^{\arcsin d} \sin ^{\alpha+1} \vartheta d \vartheta>0 \text { for } d \in(0,1) .
$$

Plugging (3.14) into (3.13) we get

$$
y=f(\lambda)=h+x_{0}^{\alpha+1} b_{\alpha}(d), \text { with } b_{\alpha}(d)=-\frac{\varphi_{\alpha}(d)}{d^{\alpha+1}}<0 \text { for } d \in(0,1) .
$$

Moreover, the volume constraint $\int f=v$ reads

$$
v=\left(h+x_{0}^{\alpha+1} \sigma_{\alpha}(d)\right) x_{0}-\frac{x_{0}^{\alpha+2}}{d^{\alpha+2}} \int_{0}^{d} \int_{0}^{\arcsin t} \sin ^{\alpha+1} \vartheta d \vartheta d t,
$$

and we write $v=h x_{0}+x_{0}^{\alpha+2} G_{\alpha}(d)$, with

$$
\begin{aligned}
G_{\alpha}(d) & =\frac{1}{d^{\alpha+2}}\left(d \int_{0}^{\arcsin d} \sin ^{\alpha+1} \vartheta d \vartheta-\int_{0}^{d} \int_{0}^{\arcsin t} \sin ^{\alpha+1} \vartheta d \vartheta d t\right) \\
& =\frac{1}{d^{\alpha+2}} \int_{0}^{d} \int_{\arcsin t}^{\arcsin d} \sin ^{\alpha+1} \vartheta d \vartheta d t>0 \text { for } d \in(0,1)
\end{aligned}
$$

The functional

$$
F\left(d, x_{0}\right)=x_{0}^{\alpha+2} G_{\alpha}(d)+h x_{0}-v .
$$

defines implicitly the constraints of the problem. Existence of a minimizer together with the Lagrange Multipliers theorem imply that there exists $\mu \in \mathbb{R}$ such that $\nabla p\left(d, x_{0}\right)=\mu \nabla F\left(d, x_{0}\right)$, namely

$$
\left\{\begin{array} { l } 
{ \partial _ { d } p = \mu \partial _ { d } F } \\
{ \partial _ { x _ { 0 } } p = \mu \partial _ { x _ { 0 } } F } \\
{ F ( d , x _ { 0 } ) = 0 }
\end{array} \Longleftrightarrow \left\{\begin{array}{l}
g_{\alpha}^{\prime}(d) x_{0}^{\alpha+1}=\mu x_{0}^{\alpha+2} G_{\alpha}^{\prime}(d) \\
(\alpha+1) x_{0}^{\alpha} g_{\alpha}(d)=\mu\left((\alpha+2) x_{0}^{\alpha+1} G_{\alpha}(d)+h\right) \\
x_{0}^{\alpha+2} G_{\alpha}(d)+h x_{0}-v=0
\end{array}\right.\right.
$$

Recalling the definitions of $g_{\alpha}$ and $G_{\alpha}$ in (3.12) and (3.16), we write the expressions for the derivatives

$$
\begin{aligned}
g_{\alpha}^{\prime}(d) & =-\frac{\alpha+1}{d^{\alpha+2}} \int_{0}^{\arcsin d} \sin ^{\alpha} \vartheta d \vartheta+\frac{1}{d \sqrt{1-d^{2}}} \\
G_{\alpha}^{\prime}(d) & =-\frac{\alpha+2}{d^{\alpha+3}} \int_{0}^{d} \int_{\arcsin t}^{\arcsin d} \sin ^{\alpha+1} \vartheta d \vartheta+\frac{1}{\sqrt{1-d^{2}}}
\end{aligned}
$$

A straightforward computation implies that for $\alpha=0,1$ we have

$$
g_{\alpha}^{\prime}(d)=d G_{\alpha}^{\prime}(d)
$$


or, equivalently:

$$
\begin{aligned}
0 & =\frac{1}{d^{\alpha+2}}\left\{(\alpha+2) \int_{0}^{d} \int_{\arcsin t}^{\arcsin d} \sin ^{\alpha+1} \vartheta d \vartheta d t-(\alpha+1) \int_{0}^{\arcsin d} \sin ^{\alpha} \vartheta d \vartheta\right\}+\frac{1-d^{2}}{d \sqrt{1-d^{2}}} \\
& =(\alpha+2) G_{\alpha}(d)-\frac{1}{d}(\alpha+1) g_{\alpha}(d)+\frac{1}{d} \sqrt{1-d^{2}} .
\end{aligned}
$$

From (3.18), the first equation in system (3.17) gives

$$
\mu=\frac{g_{\alpha}^{\prime}(d)}{x_{0} G_{\alpha}^{\prime}(d)}=\frac{d}{x_{0}}=\frac{1}{\lambda} .
$$

Plugging $\mu$ into the second equation of (3.17) we obtain

$$
(\alpha+1) x_{0}^{\alpha+1} g_{\alpha}(d)=d\left\{(\alpha+2) x_{0}^{\alpha+1} G_{\alpha}(d)+h\right\},
$$

hence, using (3.19),

$$
x_{0}^{\alpha+1}=\frac{d h}{(\alpha+1) g_{\alpha}(d)-d(\alpha+2) G_{\alpha}(d)}=\frac{d h}{\sqrt{1-d^{2}}} .
$$

We are left to compute $y=f(\lambda)=f\left(x_{0} / d\right)$ with $x_{0}=x_{0}(h, d)$ given by (3.20). Expression 3.15) for $y$, combined with 3.20 gives

$$
\begin{aligned}
y & =f\left(\frac{x_{0}}{d}\right)=h+x_{0}^{\alpha+1} b_{\alpha}(d)=h-\frac{d h}{\sqrt{1-d^{2}}} \frac{\varphi_{\alpha}(d)}{d^{\alpha+1}} \\
& =-\frac{h}{d^{\alpha} \sqrt{1-d^{2}}}\left\{\varphi_{\alpha}(d)-d^{\alpha} \sqrt{1-d^{2}}\right\}
\end{aligned}
$$

which concludes the proof.

Remark 3.6. Let $\alpha \geq 0$ and $E=\left\{(x, y) \in \mathbb{R}^{2}:|y|<f(|x|)\right\}$ be a regular minimizer of Problem (1.4) with $f$ as in (3.10). We deduce by (3.11) that $y=0$ if and only if $\alpha=0$. In fact, for any $\alpha \geq 0$ the function $d \mapsto \varphi_{\alpha}(d)-d^{\alpha} \sqrt{1-d^{2}}$ is 0 at $d=1$ and, if $\alpha>0$, it is strictly monotone decreasing since

$$
\left(\varphi_{\alpha}(d)-d^{\alpha} \sqrt{1-d^{2}}\right)^{\prime}=-\frac{d^{\alpha+1}}{\sqrt{1-d^{2}}}-\alpha d^{\alpha-1} \sqrt{1-d^{2}}+\frac{d^{\alpha+1}}{\sqrt{1-d^{2}}}<0 .
$$

Hence if $\alpha>0, \varphi_{\alpha}(d)-d^{\alpha} \sqrt{1-d^{2}}>0$ for $0<d<1$. In particular, $y<0$. On the other hand, if $\alpha=0, \varphi_{\alpha}(d)=\sqrt{1-d^{2}}$, that leads to $y=0$.

This implies that the central part of Euclidean solutions of Problem (1.4) are portions of isoperimetric sets lying in some stripe $\left\{|x|<x_{0}\right\}$, while this property fails to hold in the Grushin plane with $\alpha=1$.

\section{Appendix A. Traces of Schwarz symmetric sets}

For a set $E \in \mathscr{S}_{y}^{*}$ and a point $x_{0} \in \mathbb{R}$, the notion of trace of $E$ at $x_{0}$ can be defined thanks to the following Lemma.

Lemma A.1. Let $E \in \mathscr{S}_{y}^{*}$ and let $x_{0} \in \mathbb{R}$. Then there exist $y^{+}, y^{-} \geq 0$ such that if $T^{+}=$ $\left[-y^{+}, y^{+}\right]$and $T^{-}=\left[-y^{-}, y^{-}\right]$, there holds

$$
\lim _{x \rightarrow x_{0}^{ \pm}} \int_{\mathbb{R}}\left|\chi_{E}(x, y)-\chi_{T^{ \pm}}(y)\right| d y=0 .
$$


Proof. We prove the statement for the limit as $x \rightarrow x_{0}^{-}$. Let $u \in C^{1}\left(\mathbb{R}^{2}\right)$ and $x_{1}, x_{2} \in\left(-\infty, x_{0}\right)$. Consider the $\alpha$-gradient of $u, D_{\alpha} u=\left(\partial_{x} u,|x|^{\alpha} \partial_{y} u\right)$. We have

$$
\int_{\mathbb{R}}\left(u\left(x_{2}, y\right)-u\left(x_{1}, y\right)\right) d y=\int_{\mathbb{R}} \int_{x_{1}}^{x_{2}} \partial_{x} u(\xi, y) d \xi d y \leq \int_{\left(x_{1}, x_{2}\right) \times \mathbb{R}}\left|\partial_{x} u\right|(\xi, y) d \xi d y \leq\left|D_{\alpha} u\right|\left(\left(x_{1}, x_{2}\right) \times \mathbb{R}\right) .
$$

By the approximation theorem for $B V_{\alpha}$-functions, see [12], the last inequality can be extended to $u \in B V_{\alpha}\left(\mathbb{R}^{2}\right)$ and for $u=\chi_{E}$ we get

$$
\int_{\mathbb{R}}\left(\chi_{E}\left(x_{2}, y\right)-\chi_{E}\left(x_{1}, y\right)\right) d y \leq P_{\alpha}\left(E ;\left(x_{1}, x_{2}\right) \times \mathbb{R}\right),
$$

It hence follows that for every $\varepsilon>0$ there exists $\delta>0$, such that

$$
\left\|\chi_{E}\left(x_{2}, \cdot\right)-\chi_{E}\left(x_{1}, \cdot\right)\right\|_{L^{1}(\mathbb{R})} \leq \varepsilon \text { for } x_{0}-\delta<x_{1}<x_{2}<x_{0} .
$$

We deduce existence of a function $u \in L^{1}(\mathbb{R})$ which is the limit of $\chi_{E}(x, \cdot)$ as $x \rightarrow x_{0}^{-}$. Moreover, since for any $x \in \mathbb{R}$, the section $E_{x}=\{y \in \mathbb{R}:(x, y) \in E\}$ is a real interval centered at zero, then $u=\chi_{T^{-}}$, for a symmetric interval $T^{-}=\left[-y^{-}, y^{-}\right]$for some $y^{-}>0$.

Definition A.2 (Traces of Schwarz symmetric sets). Let $E \in \mathscr{S}_{y}^{*}$ be a set with finite $\alpha$ perimeter and let $x_{0} \in \mathbb{R}$. The interval $T^{-}$(resp. $T^{+}$) defined in Lemma A.1 is called the left (resp. right) trace of $E$ at $x_{0}$ and it is denoted by $\operatorname{tr}_{x_{0}}^{-}\left(\right.$resp. $\left.\operatorname{tr}_{x_{0}}^{+}\right)$. If

$$
\operatorname{tr}_{x_{0}-}^{x} E=\operatorname{tr}_{x_{0}+}^{x} E=\left[-y_{0}, y_{0}\right]
$$

we set $\operatorname{tr}_{x_{0}}^{x} E=\left[-y_{0}, y_{0}\right]$ and we call it the trace of $E$ at $x_{0}$ in the $x$-direction. In this case we say that the set $E$ has trace at $x_{0}$ in the $x$-direction.

Remark A.3. If $E \in \mathscr{S}_{x} \cap \mathscr{S}_{y}^{*}$ has profile function $f:[0, \infty) \rightarrow[0, \infty)$ then left and right traces at $x_{0}>0$ can be computed as:

$$
\operatorname{tr}_{x_{0} \pm}^{x} E=\left[-y_{0}^{ \pm}, y_{0}^{ \pm}\right] \text {with } \lim _{x \rightarrow x_{0}^{ \pm}} f(x)=y_{0}^{ \pm} .
$$

In fact, by definition of left and right traces, we have

$$
0=\lim _{x \rightarrow x_{0}^{ \pm}} \int_{\mathbb{R}}\left|\chi_{E}(x, y)-\chi_{\left[-y_{0}^{ \pm}, y_{0}^{ \pm}\right]}(y)\right| d y=\lim _{x \rightarrow x_{0}^{ \pm}} \mathcal{L}^{1}\left((E)_{x} \triangle\left[-y_{0}^{ \pm}, y_{0}^{ \pm}\right]\right)=2 \lim _{x \rightarrow x_{0}^{ \pm}}\left|f(x)-y_{0}^{ \pm}\right| .
$$

\section{ACKNOWLEDGMENTS}

The author would like to thank Prof. Roberto Monti from the University of Padova for suggesting her the problem and for the precious help. The author acknowledges the support of the G.N.A.M.P.A. projects

Problemi isoperimetrici e teoria geometrica della misura in spazi metrici, and Variational Problems and Geometric Measure Theory in Metric Spaces.

\section{REFERENCES}

[1] F. J. JR. Almgren, Existence and regularity almost everywhere of solutions to elliptic variational problems with constraints. Mem. Amer. Math. Soc., 165, (1976) viii+199 pp.

[2] V. Bögelein, F. Duzaar, N. Fusco, A sharp quantitative isoperimetric inequality in higher codimension. Atti Accad. Naz. Lincei Rend. Lincei Mat. Appl. 26 (2015), no. [3], 309-362.

[3] V. Bögelein, F. DuzaAr, C. Scheven, A sharp quantitative isoperimetric inequality in hyperbolic n-space. Calc. Var. Partial Differential Equations 54 (2015), no. [4], 3967-4017.

[4] L. Capogna, D. Danielli, N. Garofalo, The geometric Sobolev embedding for vector fields and the isoperimetric inequality. Comm. Anal. Geom. 2 (1994), no. [2], 203-215.

[5] A. Cianchi, N. Fusco, F. Maggi, A. Pratelli, On the isoperimetric deficit in Gauss space. Amer. J. Math., 133 (2011), no. [1], 131-186. 
[6] M. Cicalese, G. P. Leonardi, A selection principle for the sharp quantitative isoperimetric inequality. Arch. Rat. Mech. Anal. 206 (2012), no. [4], 617-643.

[7] J. Foisy, M. Alfaro, J. Brock, N. Hodges, J. Zimba, The standard double soap bubble in $\mathbf{R}^{2}$ uniquely minimizes perimeter. Pacific J. Math., 159 (1993), no. [1], 47-59.

[8] A. Figalli, F. Maggi, A. Pratelli, A mass transportation approach to quantitative isoperimetric inequalities. Inv. Math., 182 (2010) no. [1], 167-211.

[9] B. Franchi, S. Gallot, R. L. Wheeden, Sobolev and isoperimetric inequalities for degenerate metrics. Math. Ann. 300 (1994), 557-571.

[10] B. Franchi, C. E. Gutiérrez, R. L. Wheeden, Weighted Sobolev-Poincaré inequalities for Grushin type operators., Comm. Partial Differential Equations 19 (1994), no. [3-4], 523-604.

[11] B. Franchi, E. Lanconelli, Une métrique associée à une classe d'opérateurs elliptiques dégénérés. Conference on linear partial and pseudodifferential operators (Torino, 1982). Rend. Sem. Mat. Univ. Politec. Torino 1983, Special Issue, $105 Đ 114$ (1984).

[12] B. Franchi, R. Serapioni, F. Serra Cassano, Meyers-Serrin Type Theorems and Relaxation of Variational Integrals Depending Vector Fields. Houston Journal of Mathematics 22 (1996), 859-889.

[13] V. Franceschi, Sharp and quantitative isoperimetric inequalities in Carnot-Carathéodory spaces, Ph.D. Thesis, (2016).

[14] V. Franceschi, R. Monti, Isoperimetric Problem in $H$-type groups and Grushin spaces. Rev. Mat. Iberoam. 32 (2016), no. [4], 1227-1258.

[15] V. Franceschi, G. P. Leonardi, R. Monti, Quantitative isoperimetric inequalities in $\mathbb{H}^{n}$. Calc. Var. Partial Differential Equations, 54 (2015), no. [3], 3229-3239.

[16] N. Fusco, F. Maggi, A. Pratelli, The sharp quantitative isoperimetric inequality, Ann. of Math., 168 (2008), no. [3], 941-980.

[17] E. Giusti, Minimal surfaces and functions of bounded variation. Monographs in Mathematics, 80. Birkhäuser Verlag, Basel, 1984.

[18] N. Garofalo, D.-M. Nhieu, Isoperimetric and Sobolev inequalities for Carnot-Carathéodory spaces and the existence of minimal surfaces. Comm. Pure Appl. Math. 49 (1996), 1081-1144.

[19] M. Hutchings, F. Morgan, M. Ritoré, A. Ros, Proof of the double bubble conjecture, Ann. Math. 155 (2002), no. [2], $459-489$.

[20] F. MAgGi, Sets of finite perimeter and geometric variational problems. An introduction to geometric measure theory. Cambridge Studies in Advanced Mathematics, 135. Cambridge University Press, Cambridge, 2012. $\mathrm{xx}+454$

[21] R. Monti, Heisenberg isoperimetric problem. The axial case. Adv. Calc. Var. 1 (2008), no. 1, 93-121.

[22] R. Monti, D. Morbidelli, Isoperimetric Inequality in the Grushin Plane. J. Geom. Anal., 14 (2004), no. [2], 355-368.

[23] R. Monti, M. Rickly, Convex isoperimetric sets in the Heisenberg group. Ann. Sc. Norm. Super. Pisa Cl. Sci. (5) 8 (2009), no. [2], 391-415.

[24] F. Morgan, Geometric measure theory. A beginner's guide. Fourth edition. Elsevier/Academic Press, Amsterdam, 2009. viii+249 pp.

[25] P. Pansu, An isoperimetric inequality on the Heisenberg group. Conference on differential geometry on homogeneous spaces (Turin, 1983). Rend. Sem. Mat. Univ. Politec. Torino, Special Issue (1983), 159-174.

[26] D. Prandi, L. Rizzi, M. Seri, A sub-Riemannian Santaló formula with applications to isoperimetric inequalities and Dirichlet spectral gap of hypoelliptic operators, Preprint.

[27] B.W. Reichardt, Proof of the double bubble conjecture in $R^{n}$. J. Geom. Anal., 18 no. [1], (2008), $172-191$.

[28] M. Ritoré, A proof by calibration of an isoperimetric inequality in the Heisenberg group $\mathbb{H}^{n}$. Calc. Var. Partial Differential Equations, 44 (2012), no. [1-2], 47-60.

[29] M. Ritoré, C. Rosales, Rotationally invariant hypersurfaces with constant mean curvature in the Heisenberg group $\mathbb{H}^{n}$. J. Geom. Anal., 16 (2006), no. [4], 703-720.

[30] M. Ritoré, C. Rosales, Area-stationary surfaces in the Heisenberg group $\mathbb{H}^{1}$. Adv. Math. 219 (2008), no. [2], 633-671.

E-mail address: valentina.franceschi@inria.fr

Inria, team GECO \& CMAP, École Polytechnique, CNRS, Université Paris-Saclay, Palaiseau, FRANCE 